\title{
Stochastic Model Prediction of Nonlinear Creep in Glassy Polymers
}

\author{
Grigori A. Medvedev and James M. Caruthers \\ School of Chemical Engineering \\ Purdue University \\ West Lafayette, IN 47907
}

\begin{abstract}
Nonlinear creep is one of the generic features of the deformation response of glassy polymers, where a rich set of strain vs time responses is observed that depend upon the applied stress and the thermal history used to form the glass. Although existing constitutive models may be able to describe some aspects of nonlinear creep, these models are unable to provide a unified description of the diverse nonlinear creep behaviors exhibited by glassy polymer, where predicting the effects of thermal history has proved especially challenging. In this paper the ability of a recently developed Stochastic Constitutive Model (SCM) to describe nonlinear creep is critically examined, where it is demonstrated that the SCM qualitatively captures all of the major features of nonlinear creep of glassy polymers, including the tertiary creep. In particular the SCM predicts how the duration of the secondary creep and the range of the tertiary creep depend on the aging time prior to deformation. The SCM predicts the existence of Stage IV creep, which comes after the tertiary creep - where examination of nonlinear creep data clearly indicate the presence of Stage IV creep, although heretofore not recognized in the experimental data. The SCM unifies the description of nonlinear creep, and provides a detailed mesoscopic picture of the processes that underlie both strain controlled and stress controlled experiments.
\end{abstract}

\section{INTRODUCTION}

The time dependent mechanical behavior of glassy polymers is quite diverse including specific volume relaxation, nonlinear stress-strain behavior with post-yield stress softening and hardening, nonlinear stress relaxation, and nonlinear creep. Creep is important in engineering applications as well as being a probe of the nonlinear deformation processes. A typical uniaxial creep response of a glassy polymer at temperatures less than the glass transition temperature is shown in Fig. 1. The associated strain rate versus strain plot elucidates certain aspects of the response as will be discussed below. This type of creep response is observed for both thermoplastics[1-4] and thermosets.[5] Nonlinear creep is a ubiquitous feature of polymer solids and consequently a fundamental understanding of its physical origin is of considerable importance. 

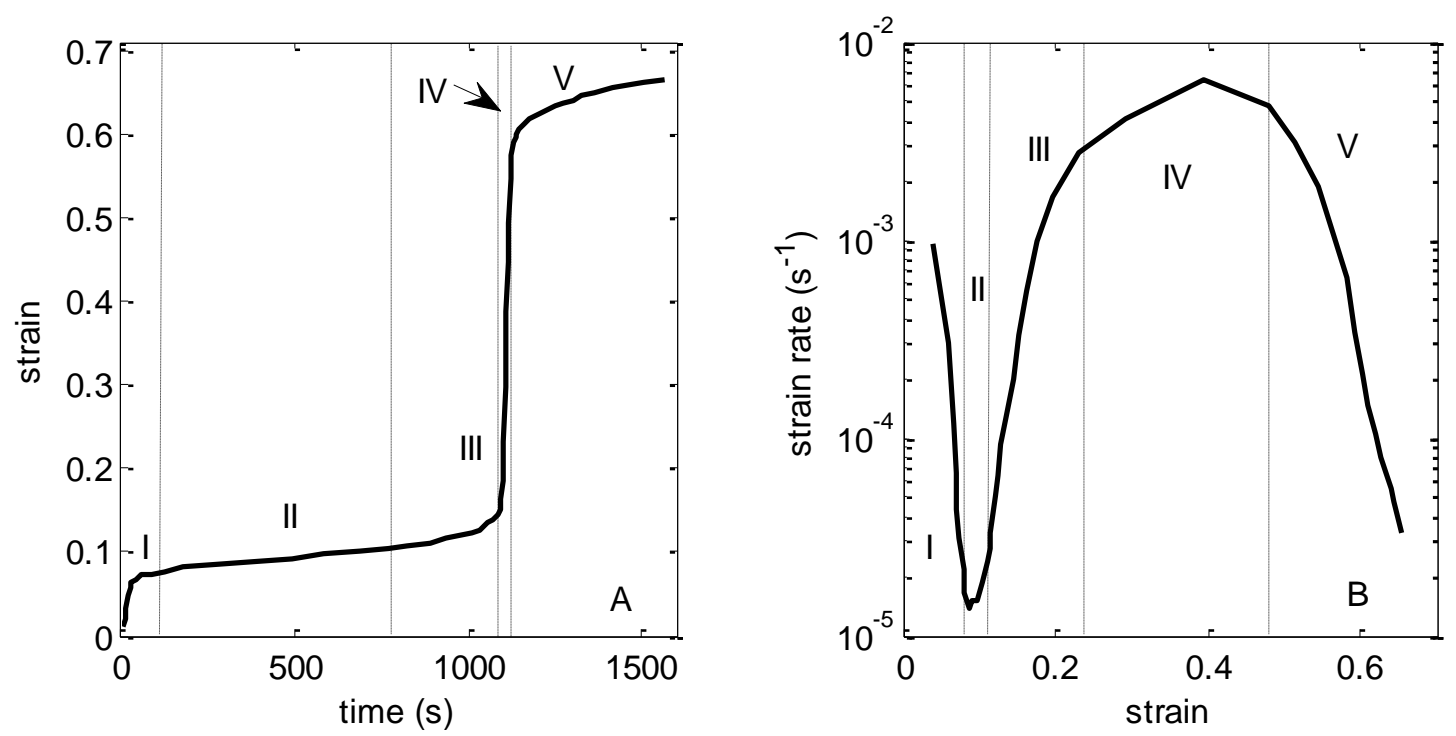

Figure 1 Creep in uniaxial compression: (A) Schematic of the various stages of nonlinear creep. (B) Strain dependence of the various stages of creep for polycarbonate at $23^{\circ} \mathrm{C}$ with an applied stress of 64MPa. (Adapted from Klompen et al.[4]).

As shown in Fig. 1 the five stages of creep are identified as: (i) primary creep where the strain rate decreases with increasing time, (ii) secondary creep where the strain rate is approximately constant, (iii) tertiary creep where the strain rate increases, (iv) a fourth stage where the strain rate is again nearly constant albeit higher than during the secondary creep, and finally (v) the fifth stage of creep where the strain rate decreases. It should be noted that this classification is not yet common as historically only Stages I and II were readily observed and some authors considered the tertiary creep as an onset of failure rather than an intrinsic material response. Even primary creep is generally non-linear, where the compliance curves for different applied stresses do not coincide. Only under sufficiently small stresses can primary and secondary creep be considered as linear viscoelastic, i.e. with the retardation spectrum obtained from creep is consistent with the relaxation spectrum from stress relaxation and with the dynamic storage and loss moduli. Both primary and secondary creep is relatively easy to observe; however, observing the full nonlinear creep response can be experimentally challenging, especially in uniaxial extension where failure often occurs at the onset of tertiary creep. All the stages of creep in uniaxial compression are clearly visible in the strain rate vs. strain data of Klompen et al.[4] for polycarbonate (i.e. Fig. 1B) as well as in the Nanzai[3] nonlinear creep data for PMMA. Ender[1] observed all stages of creep for PMMA in uniaxial extension, although the sample did exhibit necking during the Stage IV. Also, Lee et al.[5] were able to clearly observe all five stages of creep in a nominally uniaxial extension deformation for $25 \mu \mathrm{m}$ thick PMMA films as shown in Fig. 2, where optical methods were used to measure the local strain for a spatially non-uniform deformation field that exhibited necking for strains larger than $10 \%$. However, because both the axial and lateral strains were measured locally, the presence of a necked region does not affect the reported nonlinear creep data. The strain rate vs. strain data response in Fig. 2B exhibits the tertiary creep as well as the 
Stage IV and Stage V creep, although it is not possible to numerically differentiate the data in the Lee et al. paper with sufficient accuracy in order to determine the strain rate during primary and secondary creep. It has been stated that "the tertiary stage occurs at an increasing rate and terminates in fracture;"[6] however, as shown in Figs. 1 and 2 the increase in the strain rate is over a finite range of times/strains and is followed by decrease during the Stage V creep. This is an important point, because many models of the tertiary creep predict a singularity i.e. unbounded growth in the strain rate, in contradiction to the data in Figs. 1 and 2.
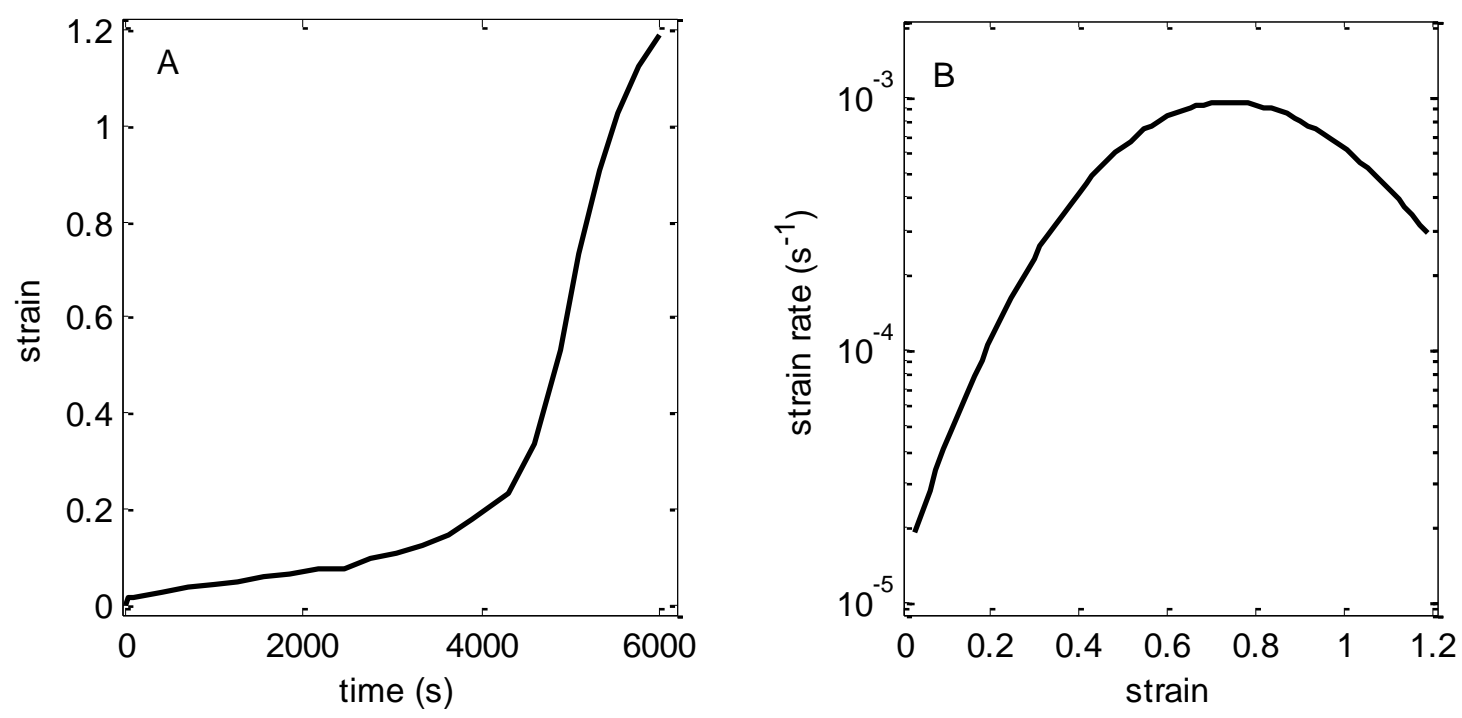

Figure 2 Strain response (A) and the associated strain rate response (B) for nonlinear creep in uniaxial extension of PMMA at 375.7K with an applied stress of 16MPa. (Adapted from Lee et al.[5])

In addition to the nonlinear creep studies shown in Figs. 1, 2 there are several other experimental measurements of nonlinear creep where at least three stages of creep have been observed, including Ender[1, 2] for PMMA shown in Fig. 3, Nanzai[3] (see Figure 2 in [3]) for PMMA, Hasan and Boyce[7] for PMMA shown in Fig. 4, and Drozdov et al[8] for poly(propylene) and poly(propylene)-clay composite shown in Fig. 5. The poly(propylene) data shown in Fig. 5 is for a semicrystalline material, where the authors argue that the deformation takes place in the amorphous phase.[8] The nonlinear creep responses shown in Fig. 5 for semicrystalline poly(propylene) are quite similar to that reported for amorphous materials in Figs. 1 through 4. For the Lee et al., Ender, Hasan and Boyce, and Drozdov et al. data, the strain vs. time curves were digitized and the corresponding strain rate vs. strain plots were created, where the results are shown in Figs. 2, 3, 4, and 5. Tertiary creep behavior in DGEBA/DEA epoxy was observed by Adolf et al.;[9] unfortunately, the reported strain versus log time data precludes numerical differentiation. The nonlinear creep behaviors shown in Figs. 1 through 5 (as well as the data in the Nanzai reference) are in qualitative agreement with each other as well as with additional experimental results to be presented in this communication (see Figs. 8 and 10 below). However, 
there are differences. Specifically, the range of increase of the strain rate during the tertiary creep varies by almost three orders of magnitude for the strain rate data in Figs. 1 and 2, etc., but only by a factor of 2.5 for some other nonlinear creep curves (e.g. Figs. 4 and 5). It is unclear whether such a significant difference in the strain rate response during tertiary creep indicates that there are different underlying deformation mechanisms or if this is just a consequence of the specific experimental conditions studied, i.e. aging time, temperature, and applied stress.
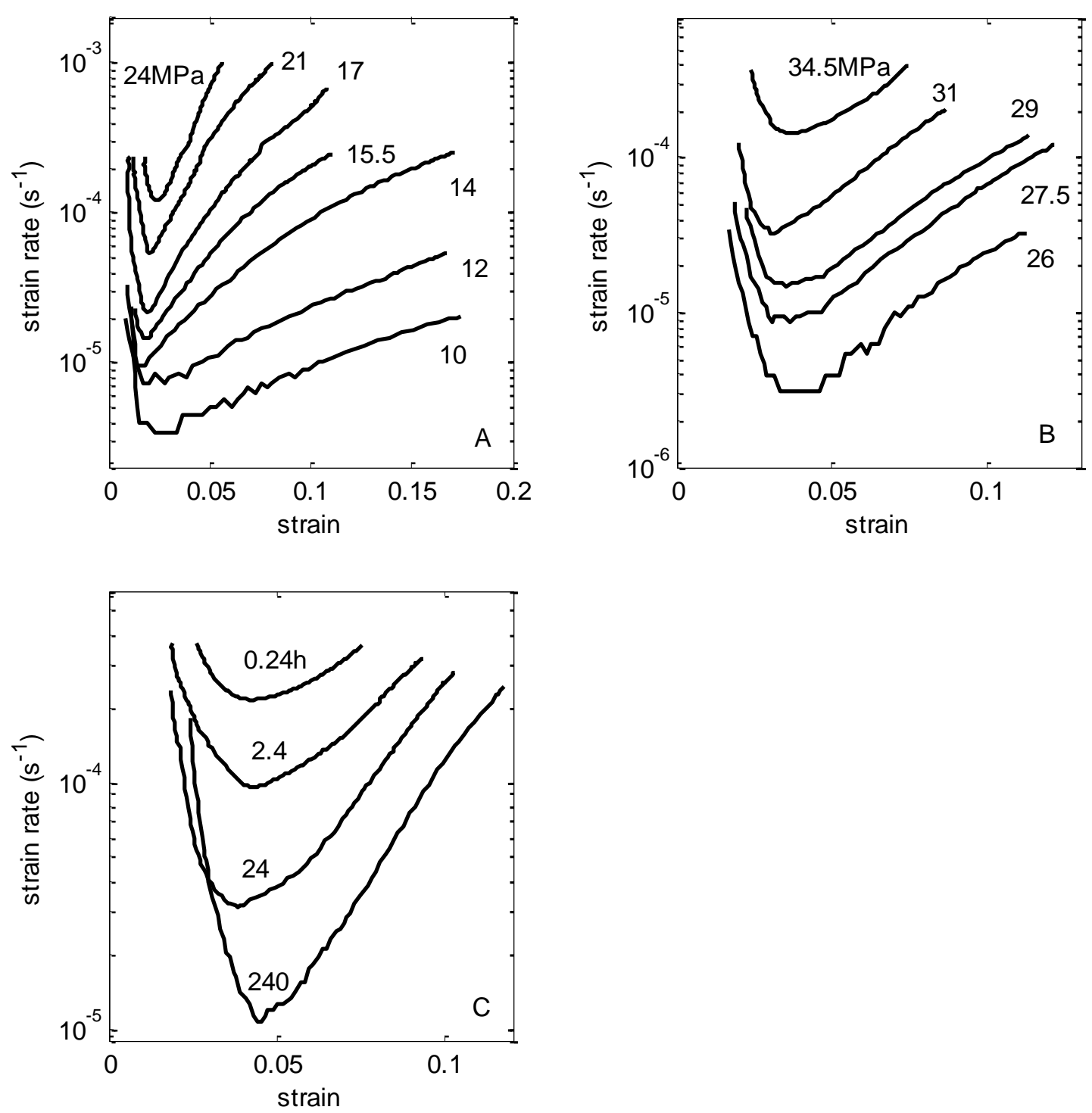

Figure 3 Strain rate vs. strain data for creep of PMMA in uniaxial extension (only the data corresponding to uniform deformation i.e. prior to necking are included) at various stresses and sub-Tg annealing times. (A) Annealed sample, temperature $-100^{\circ} \mathrm{C}\left(\mathrm{Tg}-10^{\circ} \mathrm{C}\right)$, stresses indicated in figure; (B) annealed sample, temperature $-80^{\circ} \mathrm{C}\left(\mathrm{Tg}-30^{\circ} \mathrm{C}\right)$, stresses indicated in figure; (C) sample annealed for the time indicated in figure at $100^{\circ} \mathrm{C}$, creep temperature $-80^{\circ} \mathrm{C}$, stress 27.5MPa. Data adapted from Ender[1-2]. 

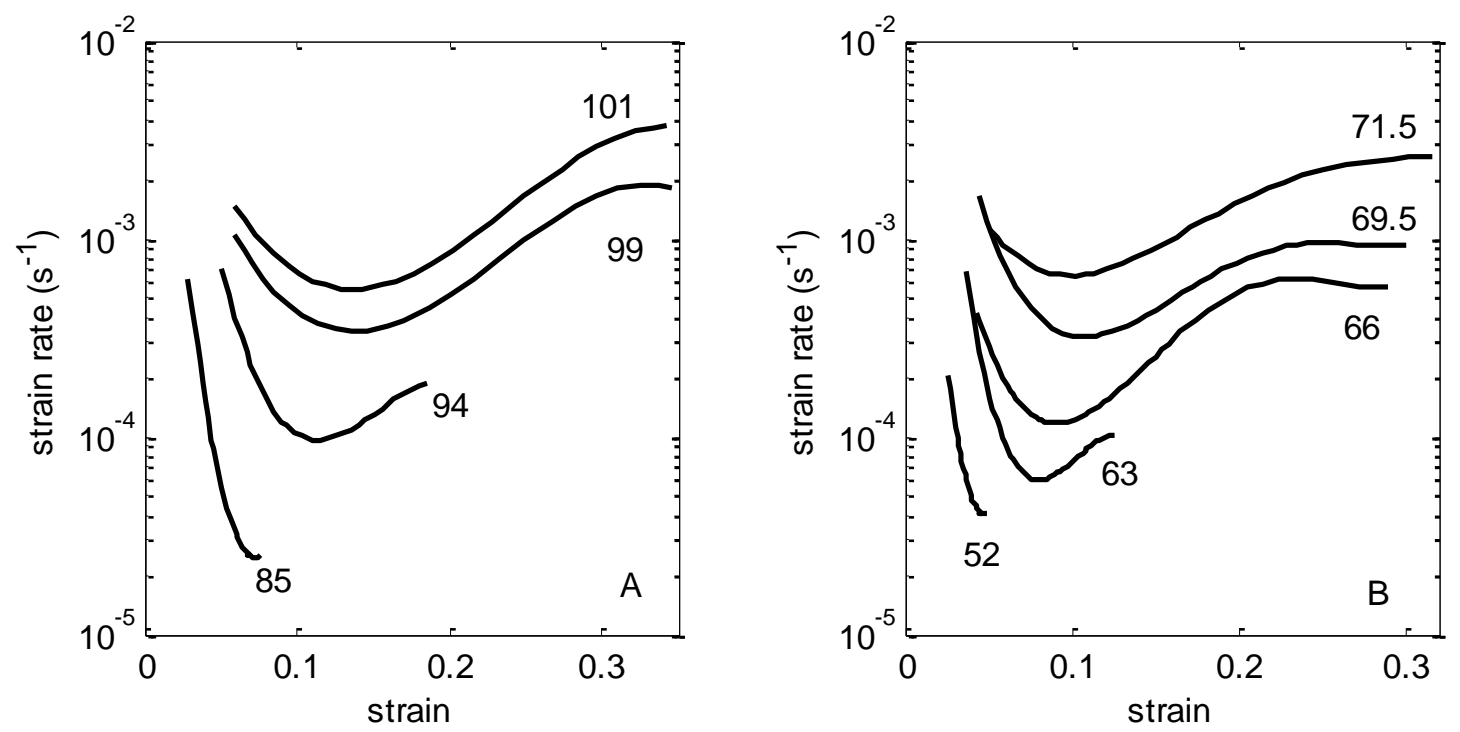

Figure 4 Creep in uniaxial compression for PMMA at (A) $23^{\circ} \mathrm{C}\left(\mathrm{T}_{\mathrm{g}}-87^{\circ} \mathrm{C}\right)$ and (B) $50^{\circ} \mathrm{C}\left(\mathrm{T}_{\mathrm{g}^{-}}\right.$ $60^{\circ} \mathrm{C}$ ). The applied axial stresses in $\mathrm{MPa}$ are indicated in figures. Data adapted from Hasan and Boyce.[7]
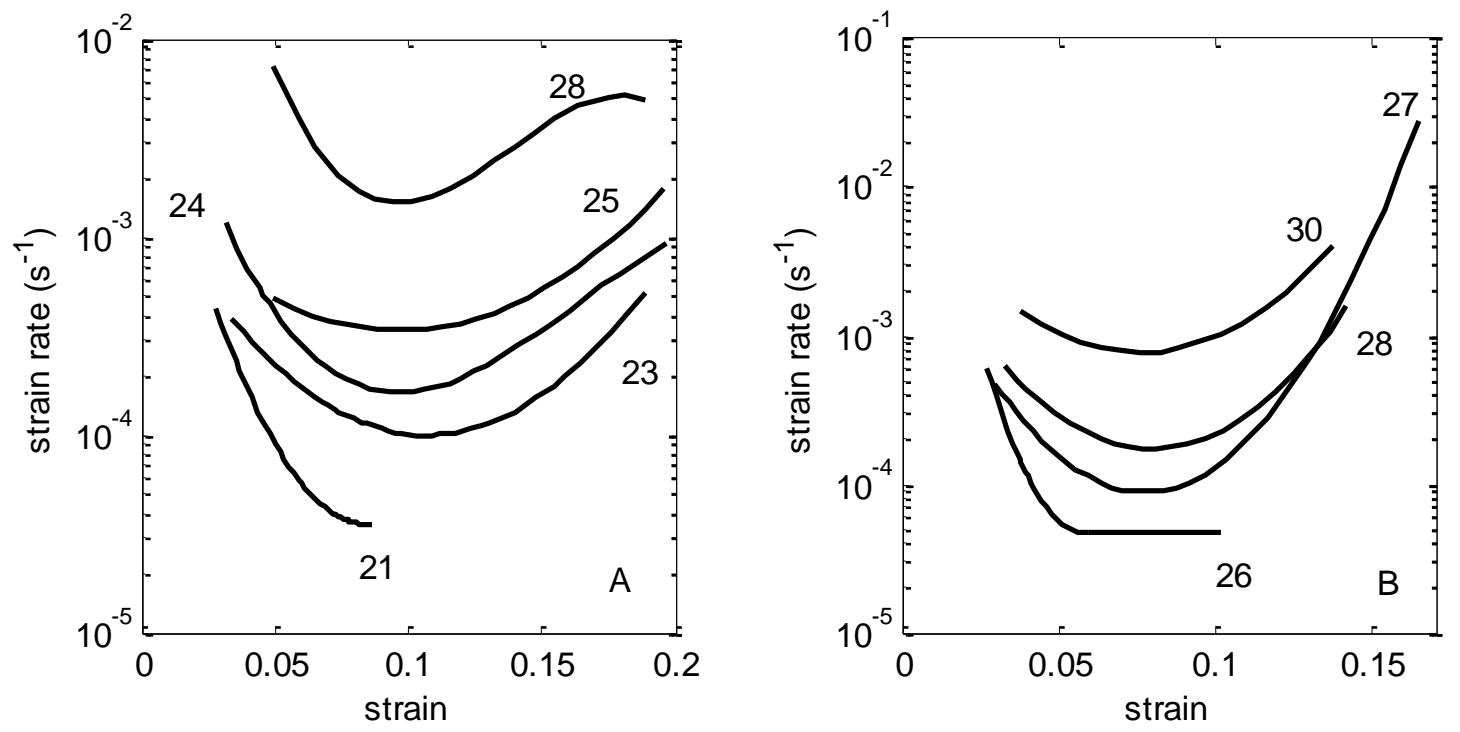

Figure 5 Creep in uniaxial extension at $23^{\circ} \mathrm{C}$ for (A) isotactic polypropylene and (B) polypropylene-clay composite. The applied axial stresses in MPa are indicated in figures. Data adapted from Drozdov et al.[8]

General features of creep in glassy polymers are:

1. The nonlinear creep behavior shown in Fig. 1 is a consistent feature of glassy polymers. 
2. The behavior shown in Fig. 1 is not due to damage. Specifically, for lightly cross-linked PMMA (see Fig. 2), the original sample dimensions as well as the tensile and shear moduli are fully recovered after Stage $\mathrm{V}$ creep, where the specimen is annealing above $\mathrm{T}_{\mathrm{g}}$. The recovery was complete even if necking occurred.[10] Nonlinear creep data for lightly cross-linked PMMA reported later in this paper also showed complete recovery after Stage IV creep.

3. The creep behavior shown in Fig. 1 is qualitatively the same in extension, compression, and shear.[11]

4. Although not the focus of this communication, the shape of the primary creep curve is affected strongly by the thermal history prior to deformation (i.e. the temperature, cooling rate, aging time, etc.) and the magnitude of the applied stress.[12]

Important observations pertaining specifically to the Stages II, III, and IV of creep, which are the focus of this communication, are:

1. The range of the secondary creep is best determined as the linear portion of the strain vs time curve, if it exists. In the log strain rate vs strain plot even sizable secondary creep may appear as merely a point (see Fig. 1B).

2. At sufficiently low applied stress e.g. curve labeled $85 \mathrm{MPa}$ in Fig. $4 \mathrm{~A}$ and curve labeled $26 \mathrm{MPa}$ in Fig. 5B, the slow rate of creep reached at the end of the primary creep never increases; in other words, the secondary creep lasts an exceedingly long time and the tertiary creep is not observed.

3. To observe the tertiary creep the $\log$ (strain rate) vs strain curve must have a minimum after which the strain rate begins to increase again. The end of the tertiary creep is manifested as a leveling off or even passing through a maximum of the log strain rate vs strain curve. Such a maximum is clearly seen in Fig. 1B and Fig. 2B whereas leveling off is observed for applied stresses of $99 \mathrm{MPa}$ and $101 \mathrm{MPa}$ in Fig. $4 \mathrm{~A}$, of $66 \mathrm{MPa}, 69.5 \mathrm{MPa}$, and $71.5 \mathrm{MPa}$ in Fig. 4B, and of $28 \mathrm{MPa}$ in Fig. 5A. These data show that tertiary creep is not a singularity i.e. there is no unbounded growth in the strain rate.

4. In light of 3 to have a large tertiary creep range requires the minimum strain rate to be low and the leveling off/maximum strain rate to be high.

5. A decrease in the applied stress decreases both the minimum (i.e. the Stage II) and the maximum (i.e. the Stage IV) strain rates. However, the value of the Stage IV strain rate is affected more than the Stage II strain rate; consequently, at sufficiently low stresses the Stage IV strain rate begins to approach the Stage II strain rate and hence no tertiary creep will occur (see 2 above).

6. Based on Fig. 3C, at a given applied stress increasing the aging time decreases the depth of the minimum in the strain rate but does not affect the leveling off value, although more data are needed in order to more fully make this conclusion.

7. Molecular probe rotation measurements during creep indicate a significant increase in molecular mobility especially during the tertiary creep, where this increase can be three 
orders of magnitude as compared to the un-deformed material. Also, the instantaneous spectrum of relaxation times dramatically narrows during nonlinear creep, where in PMMA at $13^{\circ} \mathrm{C}$ below the glass transition temperature the $\mathrm{KWW} \beta$ parameter goes from 0.32 to 0.75 as the strain increases to $20 \%$.[13]

The creep curves in Figs. 1 through 5 correspond to a single-step creep with the features described above. Multi-step creep experiments like creep-recovery also exhibit a rich response; however, multi-step creep experiments are beyond the scope of this paper.

Creep in the infinitesimal stress limit is well understood and can be described using linear viscoelasticity.[14] However, when the deformation becomes nonlinear the description becomes much more difficult. Historically the majority of the modeling efforts have been focused on describing primary and secondary creep[6], where predicting (vs. just fitting the creep data to power law time dependence with a sinh stress dependence [6]) even nonlinear primary creep for large applied stresses remains a significant effort. Moreover, creep is just one of a number of nonlinear time dependent behaviors exhibited by glassy polymers, including complex volume relaxation,[15] nonlinear stress-strain[16] with post-yield softening,[17] stress memory,[18] etc. all of which depend upon the temperature, annealing time in the glass, and deformation rate. A basic requirement of any constitutive model is that it should be able to describe the diversity of mechanical responses exhibited by glassy polymers, including all the stages of creep shown in Fig. 1.

It has been hypothesized that tertiary creep is just a manifestation of the post-yield stress softening phenomenon observed in constant strain rate deformations.[3] Specifically, the 'stress softening - tertiary creep equivalence' (SSTCE) postulate assumes that the minimum in the log strain rate versus strain data at the onset of tertiary creep is "the mirror-image" of the yield point maximum in the stress-strain curve for a constant strain rate deformation (see Figs. 2 and 3 in [3]). The correspondence between the post-yield stress softening and the tertiary creep behavior, if true, has significant implications. Specifically, the magnitude of the post-yield stress softening in the constant strain rate experiments can be changed by varying the strain rate and the sub- $\mathrm{T}_{\mathrm{g}}$ aging time. As the strain rate increases (for the same thermal history), both the yield stress and the flow stress increase, where the yield stress increases more than flow stress so that the magnitude of the stress overshoot becomes larger.[19] Also, as the aging time prior to deformation increases (at a fixed strain rate) a larger stress overshoot is observed.[20] The effect of strain rate in a constant strain rate experiment is approximately equivalent to using different applied stresses in a creep experiment, where higher applied stresses produce higher rates of creep. Of course, SSTCE cannot be perfect, since the strain rate is not constant during the creep experiment. Nevertheless, according to the SSTCE postulate one expects to see a pronounced tertiary creep behavior at higher applied stresses. The experimental data as summarized in points 2 through 5 above seem to support this hypothesis. The effect of annealing time with respect to the SSTCE postulate is revealing. For a short sub-Tg annealing time only a small stress overshoot in a constant strain rate experiment is observed, which would predict that there will only be slight tertiary creep. In 
contrast, for a long aging time a large stress overshoot is observed in a constant strain rate experiment, where the SSTCE postulate predicts pronounced tertiary creep. These predictions are in qualitative agreement with the data. In light of the importance of this conclusion we carried out a limited study of the aging time effect on tertiary creep in PMMA (see Fig. 10), which also supports the SSTCE postulate.

The focus of this communication is to (i) critically assess the ability of traditional constitutive models to describe Stages III and IV of creep and (ii) evaluate the ability of a recently developed Stochastic Constitutive Model (SCM)[21] to describe Stages III and IV of creep. The SCM has shown promise in being able to at least qualitatively predict a number of nonlinear relaxation phenomena: complex volume relaxation including the tau-effective expansion gap,[22] the nonlinear stress-strain curve including post-yield stress softening and its dependence upon annealing time,[21] nonlinear stress relaxation,[23] and stress memory[18] - phenomena that have been difficult to describe using the traditional constitutive models. If the description of nonlinear creep can be added to this set of constitutive predictions, the SCM will be a step towards a unified description of the nonlinear thermo-mechanical behavior of glassy polymers.

The rest of the paper is organized as follows: First, we review the state-of-the-art in traditional constitutive modeling of the Stages III and IV of creep in glassy polymers. We believe that since stress and strain are inherently tensorial quantities, a description of the nonlinear mechanical behavior should also be tensorial; thus, the focus of this paper will be on tensorially based models. Second, we will report on a limited experimental study of the tertiary creep in PMMA, where the effect of aging time on tertiary creep has been investigated. Third, we will present the SCM and its qualitative prediction of nonlinear creep, where the derivation of the governing SCM equations has been reported previously.[21] Finally, we will discuss the implications of the SCM prediction of the tertiary creep and the associated deformation mechanism.

\section{TRADITIONAL CONSTITUTIVE MODELING OF TERTIARY CREEP}

A number of empirical models have been used to just describe nonlinear creep.[6] An early model to describe nonlinear creep by Leaderman et al.[24] assumed that the time dependent axial strain $\varepsilon_{a}(t)$ following the instantaneous application of stress $\sigma_{a}$ is given by

$$
\varepsilon_{a}(t)=\sigma_{a} D(t)+\sigma_{a}^{2} D^{2}(t)+2 / 3 \sigma_{a}^{3} D^{3}(t)+\ldots
$$

where $D(t)$ is the linear viscoelastic compliance. Irrespective of the success or failure of the expansion given by Eqn. (1) in describing nonlinear primary creep, the Leaderman model is unable to predict tertiary creep, because the linear viscoelastic compliance is always concave downward. Using the Green-Rivlin formalism for the multi-integral expansion of the strain history functional,[25] even more general expressions can be obtained, where the time-dependent 
functions in each term are not limited to the linear viscoelastic compliance (see Findley and coworkers.[6]) However just like Eqn. (1), if the generalized relaxation kernels are concave downward, this approach will not describe tertiary creep with its concave upward shape. In addition to the difficulties of these specially developed creep models in describing Stages III and IV of creep, these models are also unable to describe the other nonlinear relaxation behaviors exhibited by polymer glasses e.g. strain control experiments.

Linear viscoelasticity qualitatively describes both primary and secondary creep but not tertiary creep, where significant nonlinearity is needed. It is straightforward to obtain tertiary creep-like behavior by simply postulating that the relaxation time is a sharply decreasing function of strain, where Ediger and coworkers dye rotation data suggests that the relaxation time decreases by three orders of magnitude when the strain reaches 50\%. However, employing a simple strain dependence of the rate of relaxation in a constitutive model will have undesirable consequences when applied to a material in the rubbery state. Specifically, the predicted relaxation spectrum of undeformed rubber specimen would differ by three orders of magnitude compared to the relaxation spectrum associated with a $50 \%$ axial strain - which contradicts experiments. A "solution" to this conundrum is to postulate that different expressions for the strain dependence of the relaxation time are applicable in the rubber vs. glassy state; however, this type of approach is little more than an empirical parameterization of experimental data - more sophisticated models that more completely acknowledge the underlying physical processes are needed.

A variety of viscoelastic and viscoplastic constitutive models have been developed to describe various aspects of the nonlinear time dependent behavior of glassy polymers. These models use a material time (or equivalently a nonlinear viscosity in the viscoplastic models) that depends upon temperature and some measure of the deformation. Material clock constitutive models that claim to predict nonlinear creep behavior including the tertiary creep include: (i) Hasan and Boyce,[7] (ii) the Eindhoven EGP model,[4,20] (iii) the Drozdov et al[8] viscoplastic-viscoelastic model, (iv) the Fielding, Larson and Cates [26] model, (v) the non-linear thermo-viscoelastic model developed by Caruthers et al.[27] which was applied to creep by Adolf et al.[9] and (vi) the scalar model of Chen and Schweizer [28]. These models will now be discussed in more details.

Viscoplastic Model of Hasan and Boyce In the Hasan-Boyce constitutive model[7] the plastic shear strain rate $\dot{\gamma}_{p}$ depends upon the shear stress $\sigma_{12}$ and three internal variables according to

$$
\dot{\gamma}_{p}=\dot{\gamma}_{0}\left[\exp \left(\frac{\sigma_{12}}{\sigma_{0}}\right)-\exp \left(-\frac{\sigma_{12}}{\sigma_{0}}+\tilde{s}\right)\right] \exp (-\tilde{a}) y\left(\frac{1}{\tilde{\alpha}}\right)
$$

where $\dot{\gamma}_{0}$ and $\sigma_{0}$ are model parameters and individual evolution equations govern the behavior of the internal variables $\tilde{a}, 1 / \tilde{\alpha}$, and $\tilde{s}$. The functional forms of $y$ and the evolution equations for $\tilde{a}$, $\tilde{\alpha}$, and $\tilde{s}$ are given in the Appendix A. The variable $\tilde{\alpha}$ is responsible for an initial hardening, which describes the transition from the primary to the secondary stage of creep; the variable $\tilde{s}$ is 
related to strain recovery upon unloading and hence is not relevant in this discussion; and, the tertiary creep behavior is due to the internal variable $\tilde{a}$ which is also responsible for the post-yield softening. The fit of the Hasan and Boyce model to the tertiary creep curves shown in [7] look good; however, there are concerns. According to the evolution equations for $\tilde{a}$ and $\tilde{\alpha}$ (See Appendix A) these variables can only monotonically decrease from the initial value to the equilibrium value and yet in Figures 14a,b in [7] they both decrease and increase. This discrepancy between the mathematical structure of the evolution equations and the reported solution of these equations calls into question the predictions of the Hasan and Boyce model.

Viscoplastic-Viscoelastic Model of Drozdov et al Drozdov et al[8] have recently proposed a constitutive model that describes tertiary creep for poly(propylene) (PP) and PP-clay composites. The model predicts the stress in terms of the strain history $\boldsymbol{\varepsilon}(t)$ (see Eqns. (15), (17), and (18) in [8]) as follows:

$$
\boldsymbol{\sigma}(t)=-p(t) \mathbf{I}+(1-\phi(t))\left[\boldsymbol{\varepsilon}_{e}(t)-\int_{0}^{t} R\left(t-t^{\prime}\right) \boldsymbol{\varepsilon}_{e}\left(t^{\prime}\right) d t^{\prime}\right] \mu
$$

where $\mu$ is shear modulus, $R$ is a material function. The strain is decomposed into elastic and plastic components, i.e. $\boldsymbol{\varepsilon}_{e}(t)$ and $\boldsymbol{\varepsilon}_{p}(t)$ respectively, and $\phi$ is an internal variable governed by the evolution equation

$$
\frac{d \phi}{d t}=a \sqrt{\frac{2}{3} \dot{\boldsymbol{\varepsilon}}: \dot{\boldsymbol{\varepsilon}}}[\Phi-\phi]^{2}
$$

with the initial condition $\phi(0)=0$; $\Phi$ is a parameter of the model with the value of 0.9 for PP. (Note that Eqn. (4) is a simplified version of Eqn. (17) in the original paper[8], where the terms omitted here for brevity do not change qualitative conclusions about the model.) In the absence of $\phi$, Eqn. (3) has a form of the conventional viscoelastic constitutive equation. The prediction of tertiary creep is due to the 1- $\phi$ factor that decreases dramatically with time during the creep experiment; however, the presence of the 1- $\phi$ factor has serious consequences for other deformation experiments. For example, consider the situation when the material is initially at rest with $\phi=0$ and then subjected to a simple uniaxial extension; the initial modulus will be $\mu$. However, if the material is first subjected to an infinitesimally small oscillatory deformation for a period of time prior to the application of the constant strain rate deformation, the value of $1-\phi$ will be much less than unity, i.e. $1-\Phi$, and the predicted initial modulus during the constant strain rate extension will be $(1-\Phi) \mu$. The infinitesimal oscillation prior to uniaxial deformation causes a significant drop in the modulus - a prediction in clear contradiction to experimental observations. There are other problems with the Drozdov model. Thus, even though the Drozdov model may be able to 'perfectly' describe tertiary creep; we believe that this is just a parameterization of a limited type of data. 
Viscoplastic-Viscoelastic Model of Kontou and Spathis Recently Kontou and Spatis[29] proposed a model which has many features in common with the model of Drozdov et al.; however, the plastic flow rule used in this model is different. Specifically, Eqn. (20) in [29] includes a scalar quantity $G$ which several steps later in the derivation is assumed to be proportional to the axial strain rate $\dot{\varepsilon}_{11}$ (see Eqns. (26) and (27) in [29]). This is not allowed in a constitutive equation, since strain rate is a tensor and can only enter as an appropriate invariant and not just as a single component of the strain tensor. Also and more importantly, the model of Kontou and Spathis predicts that tertiary creep is a singularity (i.e., the denominator in Eqn. (28) in [29] becomes zero when strain grows sufficiently large), where the rate of strain goes to infinity at a finite strain.

Eindhoven EGP Model The EGP model[4, 20] has been used to describe mechanical behavior of poly(carbonate) well below $\mathrm{Tg}$ (mostly at $23^{\circ} \mathrm{C}$ ) primarily under the constant strain rate deformations. As far as the nonlinear creep behavior is concerned no EGP predictions of the strain vs time data have been published to-date; however, time-to-failure predictions were published,[4] where 'failure' is defined as onset of the tertiary creep. Therefore it can be surmised that the EGP model does indeed predict tertiary creep, if not perhaps exact shape of the strain vs time curve. Moreover the possibility of the tertiary creep is implied by the structure of the EGP. The key nonlinearity in the EGP constitutive model is in the flow rule relating the effective rate of plastic strain $\dot{\bar{\gamma}}_{p}$ to the effective deviatoric driving stress $\bar{\sigma}_{d}^{\prime}$ as given by

$$
\dot{\bar{\gamma}}_{p}=\dot{\gamma}_{0} \exp \left(-\frac{\Delta U}{k_{B} \theta}\right) \sinh \left(\frac{\bar{\sigma}_{d}^{\prime}}{\sigma_{0}}\right) \exp \left\{-S_{a}(\theta) R_{\gamma}\left(\bar{\gamma}_{p}\right)\right\}
$$

where $\dot{\bar{\gamma}}_{p} \equiv \sqrt{\operatorname{tr}\left(\mathbf{D}_{p} \cdot \mathbf{D}_{p}\right)}, \mathbf{D}_{p}$ is the plastic strain rate tensor, $\theta$ is the temperature, ${ }^{1}$ $\bar{\sigma}_{d} \equiv \sqrt{\operatorname{tr}\left(\boldsymbol{\sigma}_{d}^{\prime} \cdot \boldsymbol{\sigma}_{d}^{\prime}\right)}$ is the effective driving stress, $\boldsymbol{\sigma}_{d}^{\prime}=\boldsymbol{\sigma}_{d}-1 / 3 \operatorname{tr}\left(\boldsymbol{\sigma}_{d}\right)$ is the deviator of the stress tensor, $\Delta U$ and $\sigma_{0}$ are model parameters, and $k_{B}$ is the Boltzmann constant. The last exponential factor in Eqn. (5) has been appended to the traditional Eyring model[30] in order to describe the post-yield stress softening in a constant strain rate deformation exhibited by glassy polymers. The EGP model contains two phenomenological functions, $S_{a}(\theta)$ and $R_{\gamma}\left(\bar{\gamma}_{p}\right)$, that are needed to describe the stress overshoot during constant strain rate deformation. $S_{a}(\theta)$ parameterizes the experimentally observed dependence of the overshoot magnitude on the temperature and the aging time. $R_{\gamma}\left(\bar{\gamma}_{p}\right)$ controls the shape of the post-yield stress-strain curve for a given thermal

\footnotetext{
${ }^{1}$ We employ $\theta$ for temperature vs. $T$ in order to be consistent with the continuum mechanics literature, where $\boldsymbol{T}$ is the stress tensor. The only exception is $T_{\mathrm{g}}$ for the glass transition temperature.
} 
history; specifically, for the deformation conditions shown in Fig. $1 R_{\gamma}\left(\bar{\gamma}_{p}\right)$ decreases by an order of magnitude as the effective plastic strain increases from 0 to $40 \%$.[20]

The $\exp \left\{-S_{a}(\theta) R_{\gamma}\left(\bar{\gamma}_{p}\right)\right\}$ factor in the EGP model Eqn. (5) is the origin of tertiary creep in accordance with the SSTCE postulate. The magnitude of change in the strain rate during tertiary creep is controlled by $S_{a}(\theta)$, which depends on the temperature and the aging time in the glassy state. The EGP model as outlined above predicts that the magnitude of the strain rate change during tertiary creep is the smallest for a quenched specimen and increases with sub- $\mathrm{T}_{\mathrm{g}}$ aging, where the maximum possible change in the creep strain rate at a given temperature will be observed for an equilibrate specimen. In summary, the EGP model does describe the tertiary creep and its dependence on the thermal history; however, this prediction is via the material function $S_{a}(\theta)$ that has to be empirically determined from the experimental data for each new thermal history, where no mechanism for the thermal history dependence of tertiary creep is provided.

Fielding-Larson-Cates (FLC) Model The FLC model[26] predicts the extensional creep of PMMA of Lee et al.[5] and the accompanying relaxation rate as measured optically from the rotation of a probe dye. The FLC model is similar in structure to the EGP model, where the total stress is the sum of two contributions: viscoelastic and essentially elastic, where the latter is responsible for the strain hardening effects. The critical component in the FLC model is the nonlinearity in the viscoelastic relaxation time. In the FLC model the relaxation time $\tau$ is an independent internal variable with its evolution described by an initial value differential equation, specifically in case of a spatially uniform uniaxial deformation[26]

$$
\dot{\tau}=1-\left(\tau-\tau_{0}\right) \mu|\dot{\varepsilon}|
$$

where $\tau_{0}$ and $\mu$ are model parameters and $\dot{\varepsilon}$ is the axial Hencky strain rate. Eqn. (6) is a strain rate based nonlinear constitutive model and as such it suffers from all the known disadvantages of strain rate models. Specifically,

1. Linear Viscoelastic Limit. A basic requirement of any constitutive model is that linear viscoelasticity is recovered in the limit of small deformations. However, if the relaxation time is a function of the strain rate, nonlinear behavior will be observed for infinitesimal deformations at very high frequencies in contradiction to the requirement of a linear viscoelasticity limit.

2. Physical Aging. In the absence of deformation (i.e. $\dot{\varepsilon}=0$ ) Eqn. (6) describes a monotonic increase in the relaxation time representing physical aging. However, Eqn. (6) predicts that a small dynamic deformation will arrest physical aging, where the relaxation time reaches a steady-state that depends on the frequency of the applied deformation. This is 
not observed experimentally, i.e. small oscillatory deformations do not arrest physical aging; in fact, it is one way that physical aging is studied.

3. Reloading Deformation. Experimental data show that for all strains (even large strains well into the hardening range) the modulus upon reloading is essentially the same as that during the original loading.[31] However, a model based on Eqn. (6) predicts something quite different. At the beginning of the first loading of an aged sample the predicted relaxation time is large; in contrast, after passing through the yield and the strain softening, the FLC model predicts that the relaxation time at the beginning of the reloading will be several orders-of-magnitude smaller. Thus, contrary to experiments the FLC model predicts that the response on reloading will differ dramatically from the one on first loading.

4. More Complex Deformation Histories. As multiple mechanical steps are included in the deformation history, strain rate based constitutive models encounter increasing problems. This occurs because in a first order evolution equation like Eqn. (6), the initial condition for the current step is the last point of the previous step. As an example, consider the prediction of recovery from creep shown in Fig. 1 of [26], where to fit the experimental observation of Lee et al.[5] there was an ad hoc change in the value of model parameters during recovery compared to the parameters during the initial creep. Specifically, the elastic modulus was decreased by an order-of-magnitude during creep-recovery, where the FLC model creep-recovery predictions would have been both nearly complete and instantaneous if the initial elastic modulus was employed - predictions that qualitatively disagree with the experimental data.

In summary, although the predictions of the creep data by FLC model appear to be impressive, it is the result of employing discrete changes in the parameters during the deformation. Moreover, the FLC model will have significant difficulties predicting other deformation histories as detailed above.

Thermoviscoelastic model Adolf et al.[9] modified the nonlinear thermoviscoelastic model (TVEM) of Caruthers et al.[27] to predict tertiary creep of a DGEBA/DEA epoxy. For the case of the irrotational deformations the constitutive equation for the Cauchy stress $\sigma$ is given by

$$
\begin{aligned}
\boldsymbol{\sigma}=\boldsymbol{\sigma}_{\infty}(\theta, \mathbf{H}) & +\frac{V}{V_{r e f}}\left[\Delta K \int_{-\infty}^{t} d \xi f_{K}\left(t^{*}-\xi^{*}\right) \frac{d I_{1}}{d \xi}-\Delta A \int_{-\infty}^{t} d \xi f_{A}\left(t^{*}-\xi^{*}\right) \frac{d \theta}{d \xi}\right] \mathbf{I} \\
& +\frac{V}{V_{r e f}} 2 \Delta G \int_{-\infty}^{t} d \xi f_{G}\left(t^{*}-\xi^{*}\right) \frac{d \mathbf{H}^{\prime}}{d \xi}
\end{aligned}
$$

where $\mathbf{H}$ is the Hencky strain tensor, $I_{1}=\operatorname{tr}(\mathbf{H}), \mathbf{H}^{\prime}$ is the deviatoric component of $\mathbf{H}, V_{\text {ref }}$ is the specific volume in a reference state and $\boldsymbol{\sigma}_{\infty}$ is the equilibrium stress. The coefficients $\Delta K, \Delta A$, and $\Delta G$ are prefactors related to the difference between respectively the glassy and rubbery bulk 
modulus, the thermal stress and the shear modulus (see Appendix A for details), and $\left\{f_{K}, f_{A}, f_{G}\right\}$ are linear viscoelastic memory kernels which are assumed to have the KWW form. The key nonlinearity in the TVEM is the 'material time' $t^{*}$ which is defined by

$$
t^{*}-\xi^{*}=\int_{\xi}^{t} \frac{d \zeta}{a(\zeta)}
$$

If the shift factor $\log a$ is a function of the defining variables, i.e. temperature, stress, specific volume, etc., then Eqn. (7) results in a traditional material clock, nonlinear viscoelastic constitutive model.[32,33,34] However, in the TVEM the $\log a$ depends not only on the current values of the state variables, but rather on the temperature and deformation histories i.e. it is $a$ functional of the temperature and deformation history (see Appendix A for details).

In the paper by Adolf, et al.[9] the TVEM predictions for creep were only shown for uniaxial extension. We now show in Fig. 6 the predicted creep response for both uniaxial extension and compression, using the same model parameters and the same thermal history employed in [9], i.e. the material was quenched at $200^{\circ} \mathrm{C} / \mathrm{min}$ to $23^{\circ} \mathrm{C}\left(\theta_{g}-47^{\circ} \mathrm{C}\right)$. The predictions in Fig. 6a show a qualitative difference between uniaxial extension vs. compression; specifically the TVEM predicts (i) apparent divergence in both the strain and strain rate at finite time that is stronger than the experimentally observed tertiary creep behavior; (ii) there is no tertiary creep in compression, contrary to experiments; and, (iii) creep is faster and the onset of tertiary creep is sooner for the annealed/slowly cooled sample vs. a sample that is quenched, which is also contrary to experimental observations (see Fig. 3). The reason that the TVEM model does not predict creep in compression is related to an intricate interplay of the relaxation integrals in the $\log a$ functional (see discussion in the Appendix A). We are not currently aware of an a priori reason why the TVEM cannot describe tertiary creep in both tension and compression, where perhaps readjusting the material parameters (or their functional forms) would allow the TVEM to describe tertiary creep in both extension and compression for the full range of thermal histories. However, it is worrisome that the emergence (or suppression) of the tertiary creep behavior in TVEM is highly sensitive to the details in the $\log a$ functional, while the experimental presence of tertiary creep is quite robust. 

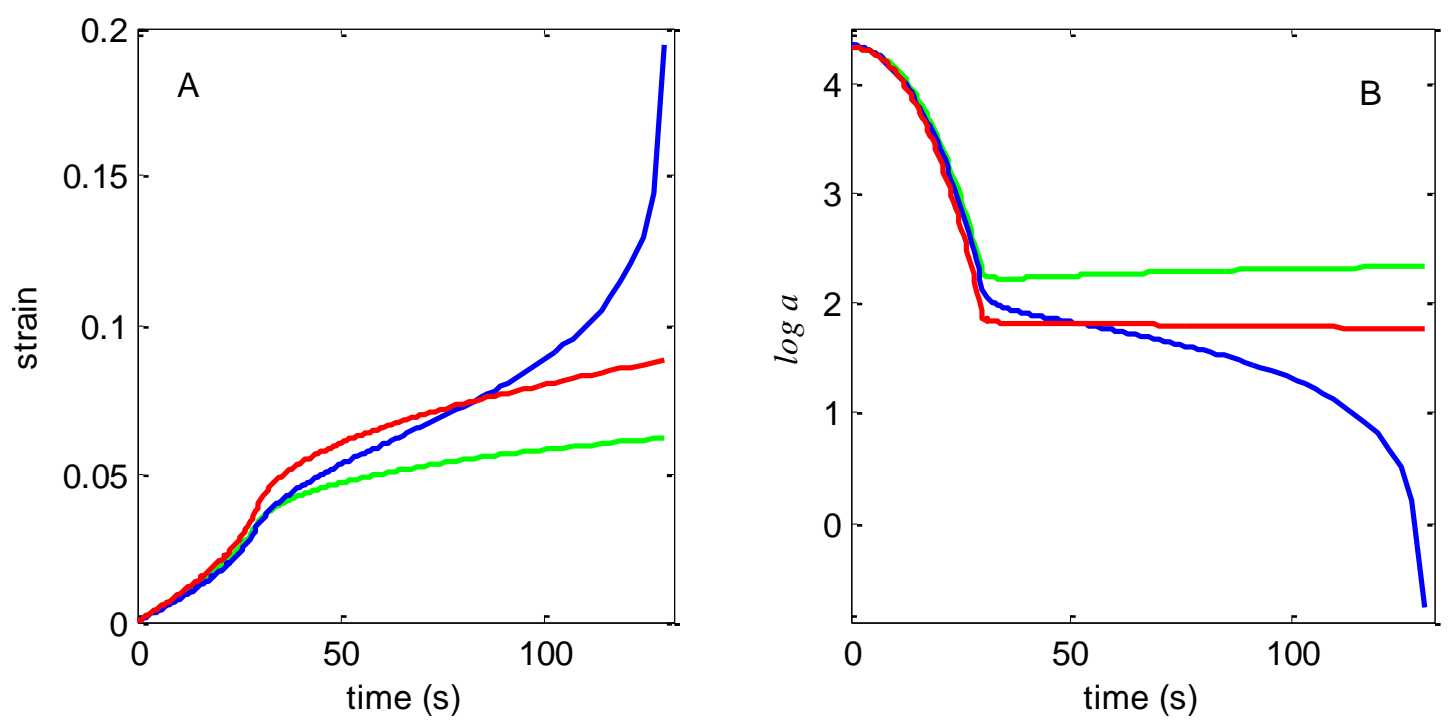

Figure 6 Predictions of (A) strain and (B) $\log a$ of the thermoviscoelastic constitutive model for an DGEBA/DEA epoxy that is quenched at $200^{\circ} \mathrm{C} / \mathrm{min}$ to $23^{\circ} \mathrm{C}$ (i.e. $\mathrm{T}_{\mathrm{g}}-47^{\circ} \mathrm{C}$ ); blue - uniaxial extension at $55 \mathrm{MPa}$ (i.e. $91 \%$ of the extension yield stress), green - compression at $63 \mathrm{MPa}$ (i.e. $91 \%$ of the compression yield stress), red - compression at $68 \mathrm{MPa}$ (i.e. $100 \%$ of the compression yield stress).

Scalar Model of Chen and Schweizer (CS) Although the focus of this paper is on tensorial constitutive models, the recent one-dimensional model of Chen and Schweizer [28] has received considerable attention and thus will be included in this summary of models. The CS model qualitatively predicts all stages of creep except Stage V, including the dependence of the Stage III creep on the applied stress and the aging time and the existence of the steady-state creep. The underlying mechanism of the Chen-Schweizer model is that the relaxation time depends on an internal variable $S_{0}$. This variable is governed by an evolution equation that includes the effects of aging and deformation, where the latter enters this evolution equation via a term that is proportional to stress squared. The CS model assumes that: (i) a single internal state variable is sufficient i.e. both aging and stress manifest through $S_{0}$ and (ii) only the average value of the internal variable matters. Because there is no distribution in $\mathrm{S}_{0}$, the width of the relaxation time distribution during creep is predicted to be constant, which disagrees with the experimental data of Lee et al. [5] The CS was able to successfully predict creep[28] and single step constant strain rate deformation, [35] but whether the single evolution equation for $S_{0}$ is able to describe other complex multi-step experiments remains an open question. In summary, the CS model is quite interesting, where the development of a tensorially correct form of the CS model and evaluation of its predictions for more complex deformation histories would be interesting. 


\section{Summary of Traditional Nonlinear Viscoelastic/Viscoplastic Continuum Models}

A number of models that have been proposed to describe nonlinear creep in glassy polymers have been critically analyzed for their ability to predict tertiary creep. The Hasan and Boyce,[7] Drozdov et al.[8] and Fielding, Larson and Cates [26] constitutive models all postulate various internal variables obeying their own evolution equations to obtain a near perfect fit to the experimental tertiary creep behavior. However, these internal variables will have adverse consequences when predicting different thermo-deformational experiments; thus, the successful description of creep is a parameterization of the experimental curves rather than a true constitutive prediction. The EGP constitutive model[20] is able to predict tertiary creep for a range of conditions, where the tertiary creep phenomenon is a result of the same mechanism that effects post-yield softening in constant strain rate experiments. However, the description of this postyield/tertiary-creep mechanism in the EGP model is purely phenomenological, where the crucial function $S_{a}(\theta)$ must be re-parameterized for each temperature and aging time. The TVEM in the form and with the parameter values used by Adolf et al.[9] predicts singularity in the creep rate which is stronger than the experimental tertiary creep in extension, but tertiary creep in compression is not predicted in contradiction to experiment. The scalar model of Chen and Schweizer [28] has made some interesting qualitative nonlinear creep predictions, but full evaluation will first require a tensorial form to be developed. The EGP and TVEM are two constitutive models that have been used extensively by the research community to describe nonlinear mechanical behavior of polymeric glasses, where these models are respectively fair representatives of the nonlinear viscoplastic and nonlinear viscoelastic approach to constitutive modeling. The fact that these constitutive models either resort to parameterization of data or are unable to provide robust predictions of tertiary creep for a range of thermal histories suggests that perhaps there is something missing in the traditional assumptions of continuum physics that underlie all of these constitutive models.

\section{EXPERIMENTAL}

The procedure for the free radical bulk polymerization of poly(methylmethacrylate), PMMA, lightly cross-linked with $1 \mathrm{wt}$. \% of ethylene glycol dimethacrylate as well as the characterization of the resulting material $\left(\mathrm{Tg}=121^{\circ} \mathrm{C} \pm 1^{\circ} \mathrm{C}\right)$ have been reported elsewhere.[36] The details of tensile stress-strain measurements using an Instron 4303 testing machine were also reported previously.[36]

Tensile creep measurements were done using an overhead loaded custom built creep apparatus, where Schaevitz DC 250DC-EC transducer was used to measure grip displacement. The apparatus compliance was determined at various stresses and temperatures by utilizing a steel strip in the clamps. The experiments took place in a forced convection oven, where the temperature was controlled to within $\pm 0.3^{\circ} \mathrm{C}$. After placing the sample in the clamps, the temperature was raised to $135^{\circ} \mathrm{C}$ and the clamps were tightened. This temperature was 
maintained for 45 minutes to remove any effects from the previous thermal/deformation history. The sample was then cooled at $1{ }^{\circ} \mathrm{C} / \mathrm{min}$ to the testing temperature and annealed at this temperature for 30 minutes before weight was added. When aging was considered, the time spent at the testing temperature before the weight was added was increased, but the rest of the procedure remained the same. Thermocouples were placed at four different locations around the sample, and thermal gradients were found to be less than $0.3^{\circ} \mathrm{C}$. Dry air was continuously flowed into the oven to prevent sample contamination with moisture.

\section{NONLINEAR CREEP BEHAVIOR OF PMMA}

The nonlinear stress-strain behavior of lightly cross-linked PMMA was measured in uniaxial extension at (i) a constant strain rate and (ii) in creep experiments. The thermal history of the samples is described in the Experimental section. The data presented in Figs. 7 and 8 are engineering stress versus engineering strain.

The goal is to focus on the experiments where the tertiary creep was observed. With this goal in mind the constant strain rate experiments are only relevant in the context of the SSTCE postulate that post-yield stress softening and the tertiary creep are related manifestations of the same underlying process. Representative examples of the effect of temperature in the $\mathrm{T}_{\mathrm{g}}-20^{\circ} \mathrm{C}$ to $\mathrm{Tg}_{\mathrm{g}}$ $10^{\circ} \mathrm{C}$ range on the stress-strain curve at fixed strain rate are shown in Fig. 7, where the yield stress values are consistent with literature data for bulk polymerized PMMA.[19] Mild post-yield stress softening was observed for all but the highest temperatures i.e. curve 1 in Fig. 7.

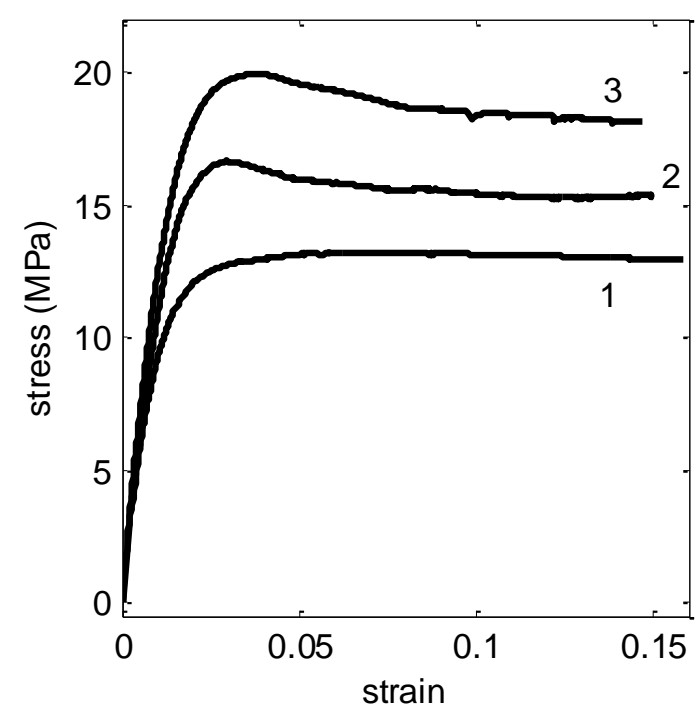

Figure 7 Stress-strain responses in uniaxial extension of PMMA stress vs. strain at a strain rate of $1.6 \cdot 10^{-4} \mathrm{~s}^{-1}$. Curve $1-110^{\circ} \mathrm{C}\left(\mathrm{Tg}-11^{\circ} \mathrm{C}\right)$; curve $2-105^{\circ} \mathrm{C}\left(\mathrm{Tg}-16^{\circ} \mathrm{C}\right)$; curve $3-100^{\circ} \mathrm{C}\left(\mathrm{Tg}-21^{\circ} \mathrm{C}\right)$. 
The results of the creep experiments for the same temperature range are shown in Fig. 8. The change in strain rate during tertiary creep is relatively small (roughly a factor of 1.6) as compared to some of the data in Figs. 1 to 5. This is in qualitative agreement with the observation that the post-yield softening in Fig. 7 is small and hence the data in Figs. 6 and 7 are consistent with the SSTCE hypothesis.
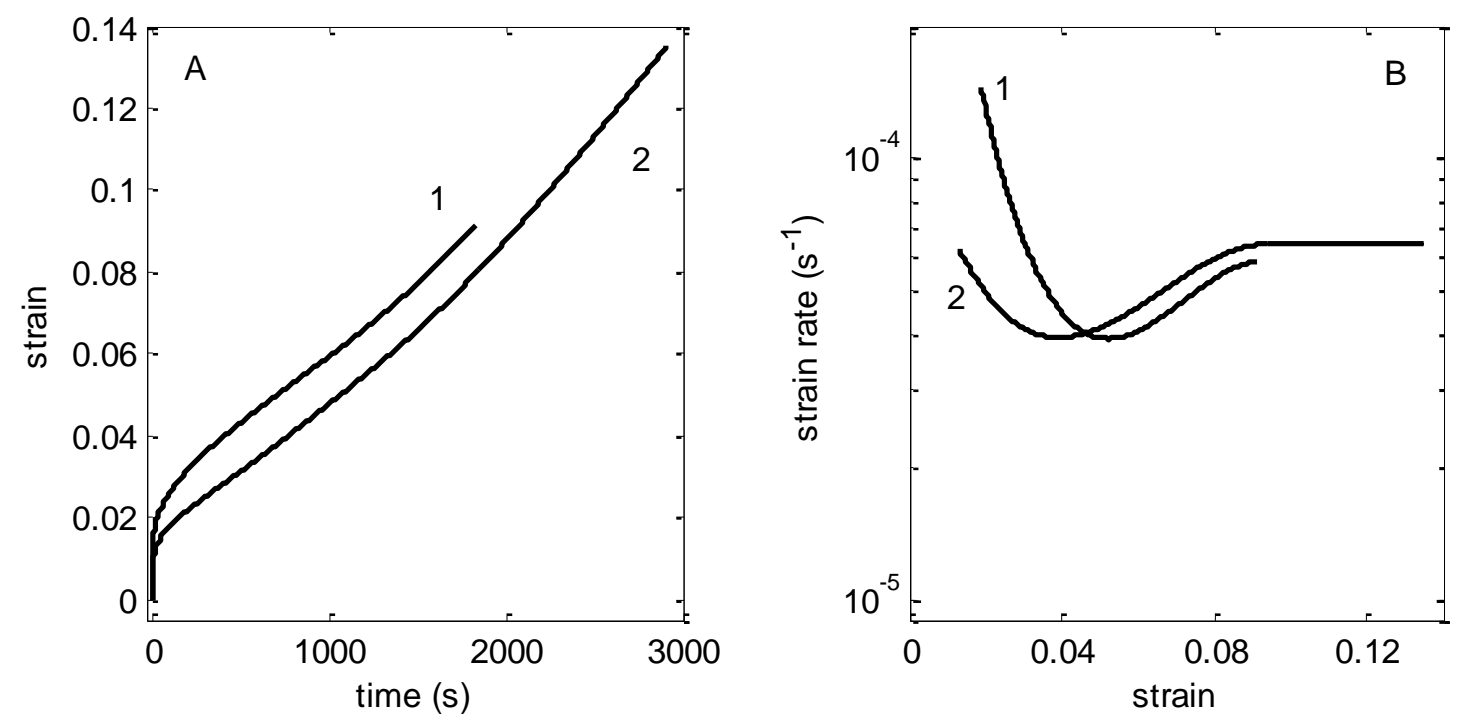

Figure 8 Uniaxial extension creep of PMMA. $1-95^{\circ} \mathrm{C}\left(\mathrm{Tg}-26^{\circ} \mathrm{C}\right)$, stress $17.9 \mathrm{MPa} ; 2-107^{\circ} \mathrm{C}$ $\left(\mathrm{Tg}-14^{\circ} \mathrm{C}\right)$, stress $12.5 \mathrm{MPa}$

A second test of the SSTCE postulate is effect of sub-Tg physical aging, where it is expected that magnitude of the creep strain rate during tertiary creep will increase with sub- $\mathrm{T}_{\mathrm{g}}$ aging since the magnitude of the stress softening increases with aging.[20] As shown in Fig. 9, the post-yield stress softening does increase; specifically, the sample aged for $0.5 \mathrm{~h}$ exhibits no stress overshoot in contrast to the sample aged for $11.5 \mathrm{~h}$ that exhibits a clearly visible post-yield softening. The results of the associated creep experiments are presented in Fig. 10, where the experimental temperature was $95^{\circ} \mathrm{C}$ (as compared to $99^{\circ} \mathrm{C}$ for the constant strain rate experiment) and the aging times were $0.5 \mathrm{~h}$ and $15.5 \mathrm{~h}$. In agreement with the SSTCE postulate, the tertiary creep shown in Fig. 10B is more pronounced in case of the aged sample, where the increase in the strain rate during tertiary creep for the $15.5 \mathrm{~h}$ aged sample is at least 4 times greater than that in the $0.5 \mathrm{~h}$ aged sample (Fig. 10B). Together with the work of Ender[2] shown in Fig. 3C this is a confirmation of a connection between the effect of sub- $T_{g}$ aging time on the tertiary creep and the effect of sub- $T_{g}$ aging time on the nonlinear stress-strain behavior. 


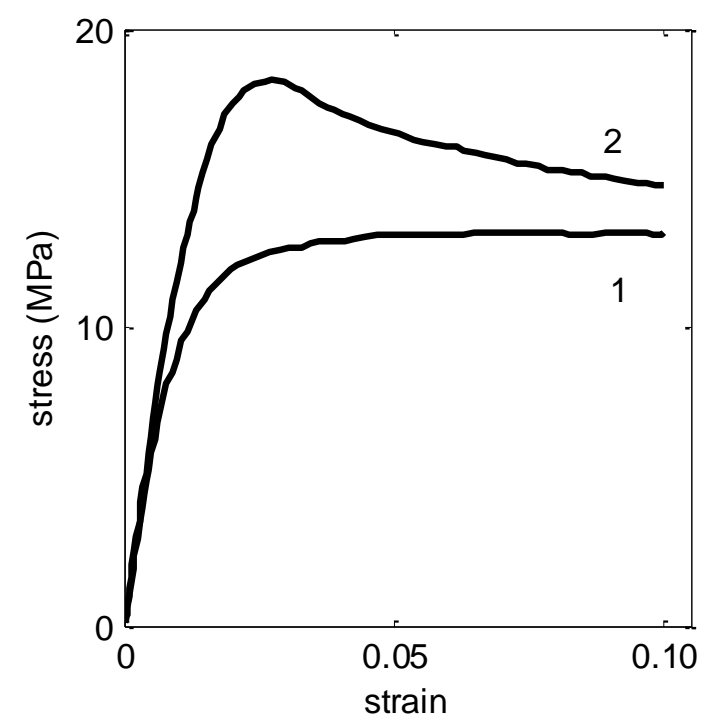

Figure 9 Effect of aging on stress vs. strain response in uniaxial extension at a strain rate of $1.6 \cdot 10^{-4} \mathrm{~s}^{-1}$ and a test temperature of $99^{\circ} \mathrm{C}\left(\mathrm{Tg}-22^{\circ} \mathrm{C}\right)$. Curve 1 - aged for $0.5 \mathrm{~h}$; curve 2-aged for $11.5 \mathrm{~h}$.
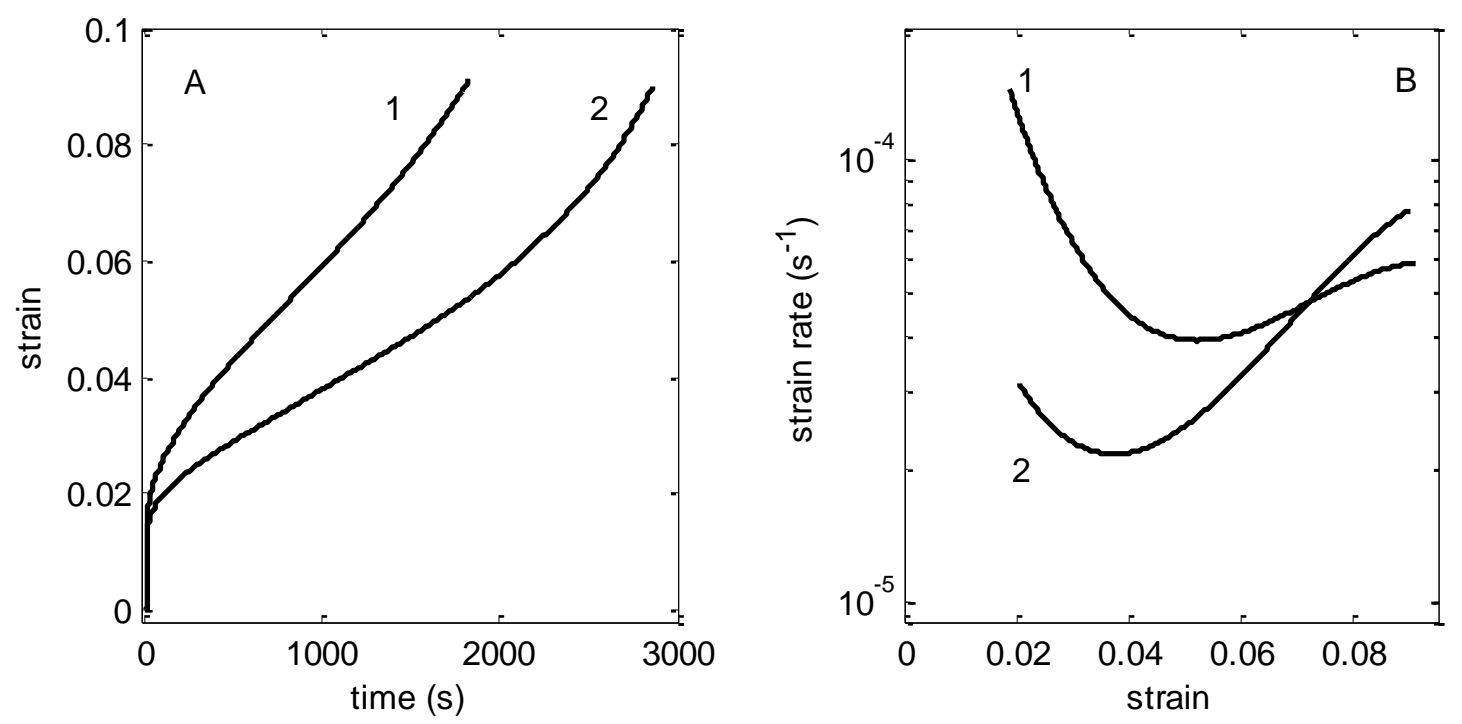

Figure 10 Effect of aging on nonlinear creep for PMMA at $95^{\circ} \mathrm{C}\left(\mathrm{Tg}-26^{\circ} \mathrm{C}\right)$ at an applied stress of $17.9 \mathrm{MPa} ; 1$ - aging for $0.5 \mathrm{~h} ; 2$ - aging for $15.5 \mathrm{~h}$. 


\section{STOCHASTIC CONSTITUTIVE MODEL PREDICTION OF THE VARIOUS STAGES OF NONLINEAR CREEP}

Traditional viscoelastic and viscoplastic constitutive models make the continuum postulate which assumes that all relevant physical quantities are spatially and temporally smooth even though it is well known that all materials exhibit significant fluctuations on the nanoscale. The key assumption in the continuum approximation is that the nanoscale fluctuations are rapid enough and symmetric enough to self-cancel and hence become irrelevant on the time scales of the physically interesting phenomena. What sets the glassy state apart is that these fluctuations, for not yet fully understood reasons, are long lived enough to manifest themselves as dynamic heterogeneities.[37] Recently a Stochastic Constitutive Model (SCM) has been developed for the description of the thermo-mechanical behavior of glassy polymers that explicitly acknowledges these long lived nanoscale fluctuations.[21] In the SCM (i) the temporal nature of the fluctuations is explicitly included, (ii) nanoscale spatial fluctuations are treated in a mean field sense and (iii) the observed macroscopic quantities are obtained as ensemble averages. The SCM approach to date has had several successes, but much remains to be done. An example of a SCM success is the prediction of post-yield softening in constant strain rate uniaxial deformations without the introduction of a new relaxation mechanism, including the dependence of the magnitude of the stress overshoot on the aging time in the glassy state.[21] The derivation of the governing stochastic differential equations of the SCM has been reported previously,[21] where the defining equations are summarized for convenience in Appendix B. The key nonlinearity in the SCM is that the mesoscopic mobility as described by the $\log \hat{a}$ shift factor depends upon the macroscopic temperature and strain as well as the fluctuating stress and fluctuating entropy, where fluctuating quantities are denoted with a 'hat'.

In the present paper the SCM model parameters already determined in [16] for a representative PMMA material will be used (see Appendix B for details). The creep response was simulated for several applied stress levels in both uniaxial extension and compression. These stress levels are chosen to be large enough to observe tertiary creep behavior within a convenient time range. To assess the magnitude of the stress associated with tertiary creep as compared to the yield stress, the constant strain rate experiments in extension and compression were simulated at a strain rate of $10^{-4} \mathrm{~s}^{-1}$, where the results are shown in Fig. 11 for two different sub- $\mathrm{T}_{\mathrm{g}}$ aging times. A definite yield response is seen, where the magnitude of the stress overshoot and the associated post-yield softening increases with sub- $\mathrm{T}_{\mathrm{g}}$ annealing. Comparing with the experimental data in Fig. 9 with the simulated response in Fig. 11, the SCM exhibits a stronger dependence of the yield stress on the annealing time than experimentally observed. However, no attempt was made in [16] to optimize the parameters of the SCM model to quantitatively fit this particular stress-strain data. As we have commented elsewhere,[23] in our opinion it is premature to optimize parameters for a limited data set until it is established that the SCM can qualitatively capture the essential features of diverse experiments. Thus, the purpose of this paper is to examine if the SCM is able to naturally capture the features of tertiary creep without introduction of new functional forms or 
specific tuning of model parameters. Consequently, we have used the SCM material parameter set from [16] without change, where the focus is on qualitative features of the SCM prediction in tertiary creep. This approach does not discount the eventual importance of quantitative fitting, but we believe that the qualitative fitting of diverse relaxation phenomena using the simplest form of the SCM needs to be completed prior to expending the considerable effort required in optimizing material parameters for a large, diverse data set.

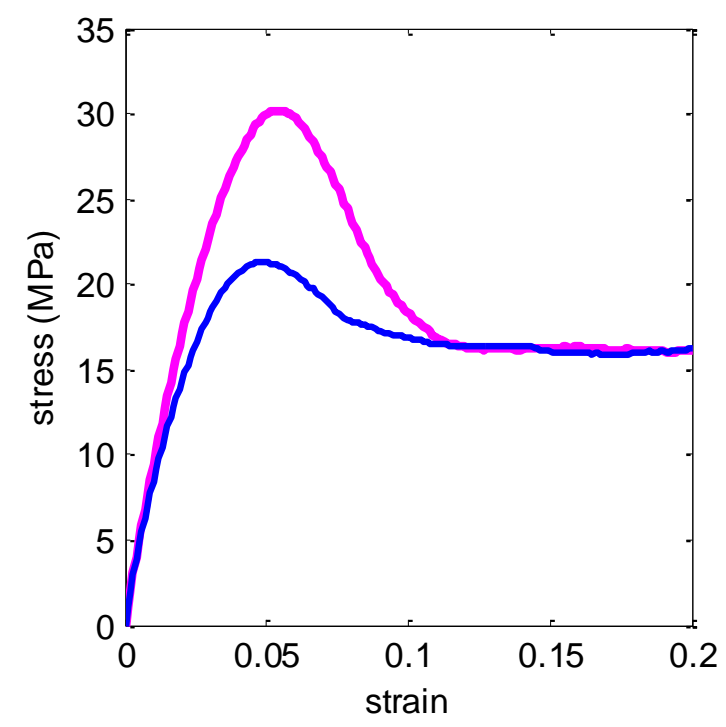

Figure $11 \mathrm{SCM}$ prediction of the stress-strain response in uniaxial extension for a constant strain rate deformation at $10^{-4} \mathrm{~s}^{-1}$ at $95^{\circ} \mathrm{C}$ following cooling from $120^{\circ} \mathrm{C}$ at $1{ }^{\circ} \mathrm{C} / \mathrm{min}$. Blue - aged for $0.2 \mathrm{~h}$; magenta - aged for $3 \mathrm{~h}$.

The creep experiment simulations for the SCM in uniaxial extension and compression are shown in Fig. 12, where one sees the transition from (i) the creep curves with just primary and secondary creep at the lowest applied stress level to (ii) curves that exhibit tertiary creep when the applied stress begins to approach the yield stress as shown in Fig. 11 for uniaxial extension. 

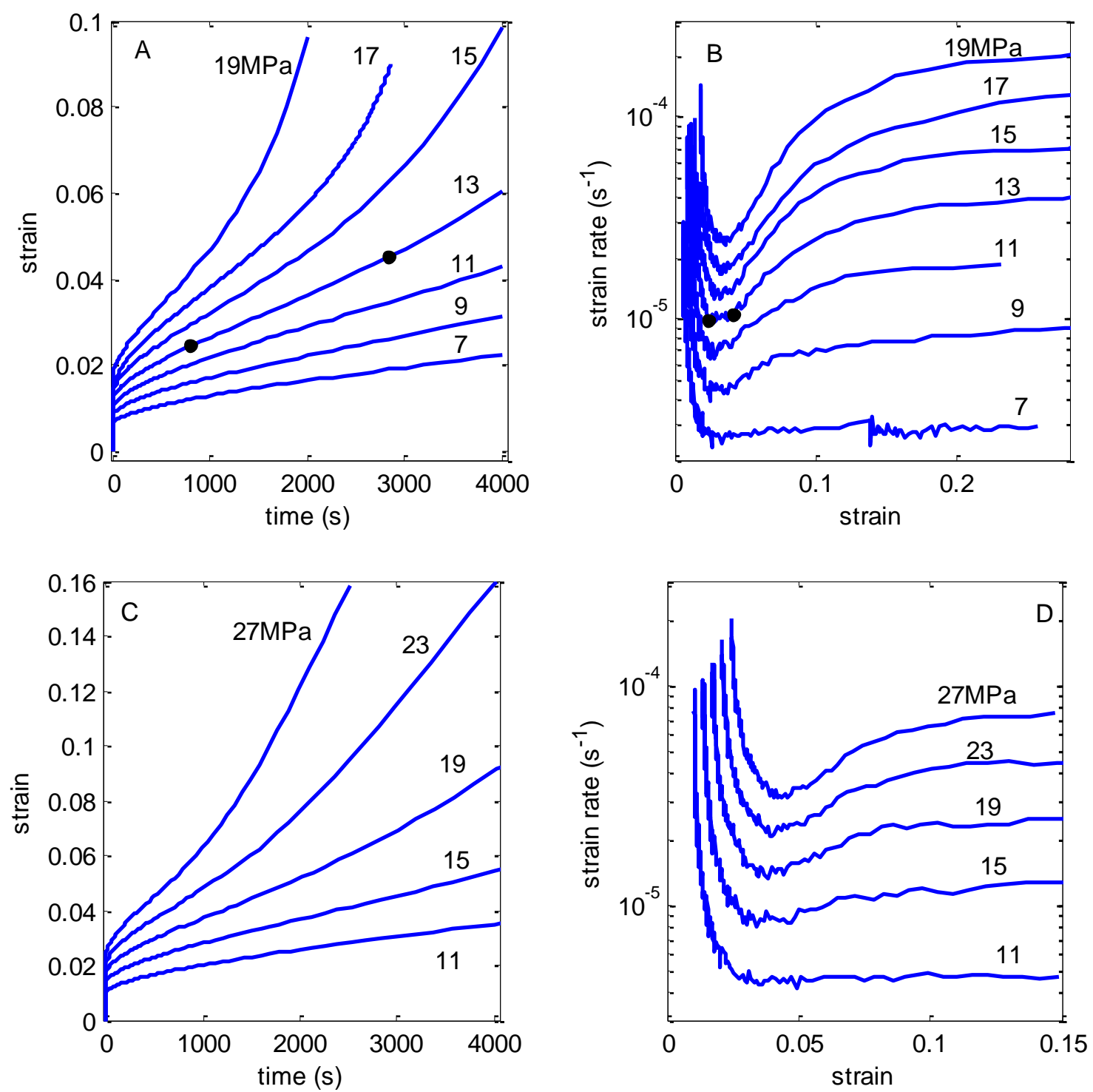

Figure 12 Stress dependence of nonlinear creep in uniaxial extension (A) and compression (C) predicted by the SCM after cooling from $120^{\circ} \mathrm{C}$ at $1{ }^{\circ} \mathrm{C} / \mathrm{min}$ to $95^{\circ} \mathrm{C}$ and aging for $3 \mathrm{~h}$. The associated log strain rate vs. strain curves are shown for uniaxial extension (B) and compression (D). The two filled circles on $13 \mathrm{MPa}$ line in (A and B) indicate the limits of the Stage II creep.

Examining the strain rate vs. strain curves shown in Fig. 12 one sees that the SCM predicts that there are two regions where the strain rate is nearly constant. The first is the region surrounding the minimum in the log strain rate vs. strain curve associated with Stage II creep, i.e. secondary creep. Even though the strain rate is not completely constant in this region, it does not undergo the dramatic changes in magnitude seen during both primary and tertiary creep. The SCM predicts there is another region of approximately constant strain rate at larger strains that begins at the end of the tertiary creep region, which we designated in the Introduction as Stage IV creep, where Stage III creep, i.e. tertiary creep, is the transition between the two regions with 
approximately constant strain rates. Examining the results in Fig. 12, the identification of secondary creep is somewhat arbitrary, where the strain rate is never truly constant. The SCM does predict that the strain rate approaches a constant at sufficiently large strains. This prediction of Stage IV creep is consistent with the creep data for PMMA and PP shown in Figs. 4A, 4B, and 5A and listed in Point 3 in the Introduction. The SCM predicts the first four stages of nonlinear creep, but it does not predict Stage V creep as shown in Fig. 1.

The SCM simulations shown in Fig. 12 were for the case of a constant axial stress, where a constant force is the more experimentally relevant condition that would account for the change in cross-sectional area with deformation. However, for the strains shown in Fig. 12 there is no measureable difference between using a constant axial stress or a constant axial force, where both boundary conditions exhibit all four stages of creep.

The evolution of the two critical internal variables of the SCM during creep is shown in Fig. 13 for the nonlinear creep experiments corresponding to the $17 \mathrm{MPa}$ (pronounced tertiary creep) and the $9 \mathrm{MPa}$ (slight tertiary creep) curves in Fig. 12A. As shown in Figs. 13A, during creep with a load of $17 \mathrm{MPa}$, the spectrum of the relaxation times (i.e. the distribution of the mesoscopic $\log \hat{a}$ ) shifts towards shorter times by one-and-a-half orders of magnitude and becomes narrower, where both of these features are in qualitative agreement with the experimental observations of Ediger and co-workers.[5, 38] Although the $\log \hat{a}$ pdf is not exactly fit by the KWW form, for the data in Fig. 13A the KWW $\beta$ parameter is 0.35 just prior to the application of the stress (i.e. the black spectrum) and increases to 0.47 during tertiary creep (i.e. the red spectrum). What controls the extent of tertiary creep is the magnitude of the change in the $\log \hat{a}$ pdf shown Figs. 13A vs 13C. Specifically, just prior to deformation the $\log \hat{a}$ pdf is given by the black curve; however, once an axial stress is applied the $\log \hat{a}$ pdf begins to evolve towards a new steady state pdf that is shown by the red curves in Figs. 13A and 13C. There is very little change in the log $\hat{a}$ pdf for a $9 \mathrm{MPa}$ applied stress and the $\log \hat{a}$ pdf just prior to application of the load as compared to the change in the $\log \hat{a}$ pdf for a $17 \mathrm{MPa}$ applied stress. Tertiary creep is a result of the transition between the initial and the applied stress dependent stationary $\log \hat{a}$ pdf, where if the difference between initial and stationary spectra is small there will be only slight tertiary creep. In other words, if the Stage IV strain rate is quite different than the strain rate during secondary creep (i.e. the minimum in the $\log$ strain rate vs. strain plot), then this significant increase in strain rate is tertiary creep. However, if the stress is such that the Stage IV creep rate is nearly the same as the minimum in the log strain rate vs. strain plot, then primary creep transitions directly to Stage IV creep and tertiary creep cannot be observed.

The key contribution to the evolution of $\log \hat{a}$ is the fluctuating axial stress $\hat{x}_{3}$, which is shown in Figs. 13B and 13D for applied axial stresses of $17 \mathrm{MPa}$ and $9 \mathrm{MPa}$, respectively. There are two distinct peaks in the $\hat{x}_{3}$ distribution - a peak centered on $\hat{x}_{3} \sim 0$ that is associated with soft/mobile domains and a peak associated with hard/rigid domains that moves towards more negative axial 
stresses as the deformation proceeds. Comparing Fig. 13B vs $13 \mathrm{D}$, the change in the $\hat{x}_{3}$ distribution during deformation is qualitatively similar for both the $17 \mathrm{MPa}$ stress that exhibited large tertiary creep and $9 \mathrm{MPa}$ stress that only exhibited slight tertiary creep. The stationary $\hat{x}_{3}$ pdf at long times from Figs. 13B and 13D are re-plotted together in Fig. 14. The 17MPa stationary distribution (red) has a more pronounced tail and a much lower height of the peak near $x_{3}=0$, where the currently "soft"/mobile meso-domains are located. The SCM does not predict any change in mechanisms as the nonlinear creep response transitions from primary to secondary to tertiary creep. This is a different perspective than that employed in other proposed constitutive models, where new relaxation mechanisms become active during tertiary creep, e.g. the plastic component of total strain starts to dominate once the yield point has been reached as is occurring in the EGP model.
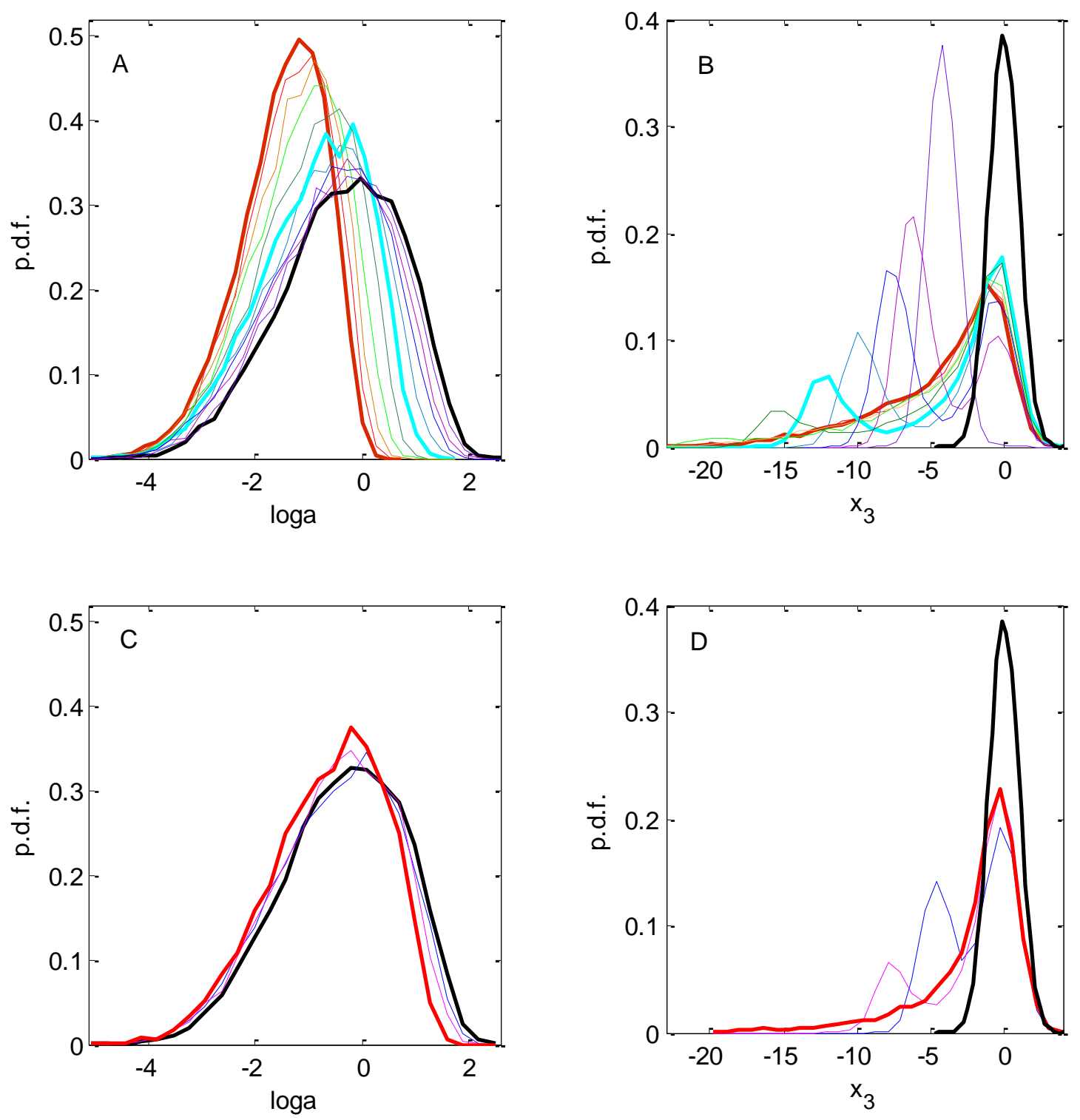
Figure 13 Evolution of the distribution of $\log \hat{a}$ and of the axial stress $\hat{x}_{3}$ in the SCM during uniaxial extension creep after annealing for $3 \mathrm{~h}$ at $95^{\circ} \mathrm{C}$ : A and $\mathrm{B}$ - the applied stress is $17 \mathrm{MPa}$; C and $\mathrm{D}$-the applied stress is $9 \mathrm{MPa}$. The pdf curves correspond to different times along the creep curves shown in Fig. 12A. Black curve - pdf prior to application of the creep load; in (A) and (B) subsequent curves the pdf moving from right to left indicate creep strains from $0 \%$ to $20 \%$ with an approximate steps of $2 \%$; red curve - pdf during final constant strain rate creep.

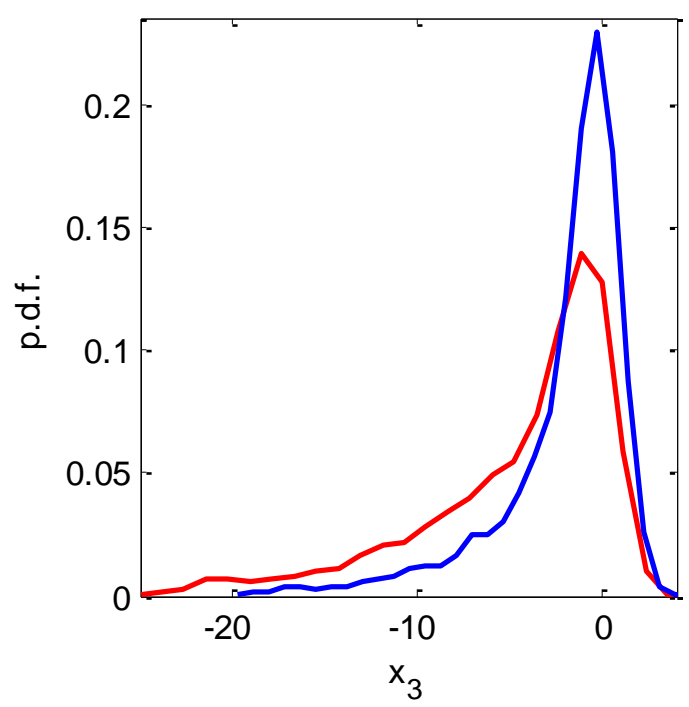

Figure 14 Stationary distribution of the axial stress $\hat{x}_{3}$ in the SCM during uniaxial creep at an applied stress of $17 \mathrm{MPa}$ - red and $9 \mathrm{MPa}$ - blue; this is re-plotting of red curves from Fig. 13B and 13D. For blue $\operatorname{pdf}\left\langle\hat{x}_{3}\right\rangle=-2.2$ and for red $\operatorname{pdf}\left\langle\hat{x}_{3}\right\rangle=-4.7$.

The origin of the evolution of the $\hat{x}_{3}$ and $\log \hat{a}$ distributions during a constant strain rate deformation has been addressed in details in [21] and [23], where we now give a brief recap necessary for understanding the SCM prediction of the tertiary creep. In the absence of deformation the meso-domains have values of $\hat{x}_{3}$ normally distributed around zero so that $\left\langle\hat{x}_{3}\right\rangle=0$ (i.e. the black curves in Fig. 13B and 13D), where $<>$ indicates an ensemble average. In this case the external/applied stress is zero, i.e. $x_{3}^{\text {ext }}=0$, and the boundary condition in the form of

$$
x_{3}^{e x t}=\left\langle\hat{x}_{3}\right\rangle
$$

is trivially satisfied. Prior to application of the external stress the pdf of the configurational entropy $\hat{x}_{0}$ is such that $\left\langle\hat{x}_{0}\right\rangle \neq 0$ unless the system was annealed long enough to reach equilibrium. In a typical experimental situation where the material has been cooled from above $\mathrm{Tg}$ to, say, $\mathrm{Tg}-25^{\circ} \mathrm{C}$ and annealed for several hours, the $\hat{x}_{0}$ entropy distribution, and hence the $\log \hat{a}$ distribution, prior to deformation is still slowly relaxing toward equilibrium. In the presence of an externally applied deformation (which can either be stress or strain controlled) the response of an 
individual meso-domain depends on its current value of $\log \hat{a}$. If $\log \hat{a}$ is small (we designate such meso-domain as 'soft') then the systematic driving by the external load term is overwhelmed by relaxation and fluctuation terms and consequently the instantaneous response is no different from the one without the load. In contrast, if $\log \hat{a}$ is large (we designate such meso-domain as 'rigid') then the systematic driving term dominates the relaxation and fluctuation terms, and the instantaneous response is that of an elastic solid. The longer a given meso-domain remains rigid, the higher absolute value of $\hat{x}_{3}$ it will attain. A meso-domain stops being rigid in two ways - (i) stochastically via a fluctuation in any or all components of $\hat{\mathbf{x}}$ (but primarily via positive fluctuation in $\hat{x}_{0}$ ) rendering its $\log \hat{a}$ small or (ii) when absolute value of $\hat{x}_{3}$ becomes so large that the corresponding $\log \hat{a}$ is small regardless of what values other components of $\hat{\mathbf{x}}$ have. Conversely, a soft meso-domain becomes rigid only stochastically, primarily via negative fluctuation in $\hat{x}_{0}$. As a result in the presence of deformation a circulation of meso-domains arises in the $\hat{x}_{0}-\hat{x}_{3}$ state space, where a freshly made rigid domain travels along the $x_{3}$ axis (in the negative direction in case of extension) only to eventually revert to being soft at a sufficiently large deformation.[23] Thus, one of the important effects of deformation is that the progress of the $\hat{x}_{0}$ distribution, and hence the $\log \hat{a}$ distribution, toward equilibrium is arrested. The presence of the two competing trends: aging i.e. shift of the $\log \hat{a}$ distribution toward longer times due to $\hat{x}_{0}$ relaxation vs. mechanical "rejuvenation" i.e. shift of the $\log \hat{a}$ distribution toward shorter times due to increase in absolute value of $\hat{x}_{3}$, leads to the existence of the steady state pdf in both $\log \hat{a}$ and $\hat{x}_{3}$ (red curves in Fig. 13, see also Fig. 14). A point of caution: in the discussion above we have used the terminology 'soft' and 'rigid'; however, this is an arbitrary distinction, where there is a continuous distribution of domains, Moreover, no domain is permanently 'soft' or permanently 'rigid', but rather is continually changing its relaxation time across the full continuum of relaxation times.

As shown schematically in Fig. 15, the emergence of the tertiary creep is determined by whether the steady-state $\log \hat{a}$ pdf with an applied stress differs significantly from the $\log \hat{a}$ pdf just prior to deformation. If the difference is significant - there is tertiary creep (i.e. compare the red vs. solid blue curves in Fig. 15 and Fig. 13A for the 17MPa deformation); if it is not - there is only slight tertiary creep (i.e. compare the green vs. solid blue curves in Fig. 15 and Fig. 13C for the 9MPa deformation). The reason for why the emergence and extent of tertiary creep depends on the applied stress value can now be clearly identified. Specifically, the boundary condition given in Eqn. (9) requires that to maintain larger absolute value of the applied stress $x_{3}^{\text {ext }}$ the steady-state $\hat{x}_{3}$ distribution must have a longer tail, i.e. for uniaxial extension this means the $\hat{x}_{3}$ pdf extends farther to the left as shown by the red curve in Fig. 14 for an applied stress of 17MPa. This means that more newly made rigid domains (with the initial values of $\hat{x}_{3}$ near zero) need to reach higher 
absolute $\hat{x}_{3}$ values, which can only be achieved by making the strain rate higher. However, the faster strain rate also means that the cutoff of the steady state $\log \hat{a}$ pdf occurs at shorter times as shown by the arrows in Fig. 15 (see [23] for a detailed explanation of origins of this strain rate dependent cut off of the $\log \hat{a}$ pdf); thus, the $\log \hat{a}$ pdf spectrum associated with the larger applied stress, i.e. red curve in Fig. 15, is shifted to the left and is consequently much different than the $\log \hat{a}$ pdf just prior to the creep deformation. When the applied axial stress is smaller (e.g. the 9MPa creep response shown in Figs. 12 and 13), the steady-state $\hat{x}_{3}$ distribution does not require a longer tail to satisfy Eqn. (9) as illustrated by the blue curve in Fig. 14. Applying the same reasoning as above, the steady state strain rate does not need to be as fast, which in turn means that there is only a slight perturbation of the initial $\log \hat{a}$ pdf as shown schematically in Fig. 15; the steady state strain rate in Stage IV creep is nearly equivalent to the strain rate at the end of primary creep. Consequently, there is no increase in strain rate and thus no appreciable tertiary creep.

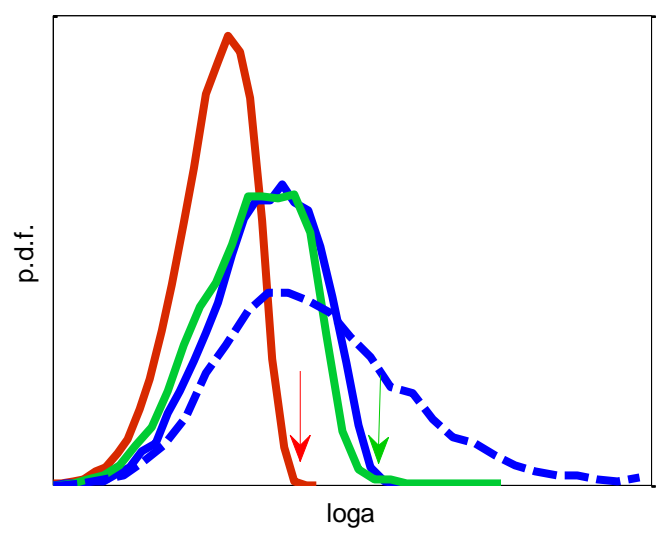

Fig. 15 Schematic $\log \hat{a}$ pdf for undeformed glass: solid blue - quenched, dashed blue completely annealed i.e. equilibrated, and the steady state $\log \hat{a}$ pdf for two different levels of creep stress: green - moderate, red - large. Arrows indicate where the $\log \hat{a}$ pdf is cut off due to the deformation.

The effect of sub-Tg aging on tertiary creep is shown in Fig. 16, where the results are in qualitative agreement with experimental findings shown in Fig. 10. Thus the SCM not only predicts the various stages of creep, but it is also able to naturally predict that for tertiary creep the range of the strain rate increase grows with sub- $\mathrm{T}_{\mathrm{g}}$ annealing. Examining Fig. 16C, the SCM predicts that for the blue curve i.e. material annealed for $0.2 \mathrm{~h}$, the log of strain rate changes from 4.27 at the minimum to -3.89 for the asymptotic stage IV creep value - a change of -3.89-($4.27)=0.38$ during tertiary creep. For the magenta curve i.e. material annealed for $3 \mathrm{~h}$, the value at the minimum is -4.78 and hence the change in the log strain rate during tertiary creep is -3.89-(4.78)=0.89. The SCM predicts half a decade (i.e. 0.89 vs 0.38) larger tertiary creep range for material annealed for $3 \mathrm{~h}$ vs $0.2 \mathrm{~h}$. This is in reasonable agreement with the experimental value of 
0.4 obtained for the magenta vs blue curve in Fig. 10B corresponding to material annealed for $15.5 \mathrm{~h}$ vs $0.5 \mathrm{~h}$. Obviously the experimental data for fully equilibrated material are not available; hence there is no black curve in Fig. 10. Numerical simulation poses no such limitation and the SCM prediction for fully annealed material is shown as black curve in Fig. 16. The difference between the minimum and the asymptote value is 1.8 in other words the strain rate is predicted to change by nearly two orders of magnitude during tertiary creep.

The annealing time dependence of tertiary creep is a direct consequence of evolution of the $\log \hat{a}$ pdf. As the material is annealed below $\mathrm{T}_{\mathrm{g}}$ prior to deformation, the $\log \hat{a}$ pdf immediately following the quench i.e. solid blue curve in Fig. 15 evolves towards the $\log \hat{a}$ pdf for a material at equilibrium shown as dashed blue curve in Fig. 15. For a fully annealed material, the equilibrium $\log \hat{a}$ pdf (i.e. the dashed blue line in Fig. 15) is very different than the steady state $\log \hat{a}$ pdf for a 17MPA applied stress (i.e. the red line in Fig. 15). Thus, the annealed material will exhibit dramatic tertiary creep as illustrated in Fig. 16B. It should be noted that the onset of this tertiary creep behavior in case of the equilibrated material is significantly delayed as compared to the material annealed for $3 \mathrm{~h}$ as evidenced by the different time range in Fig. 16B vs Fig. 16A. 

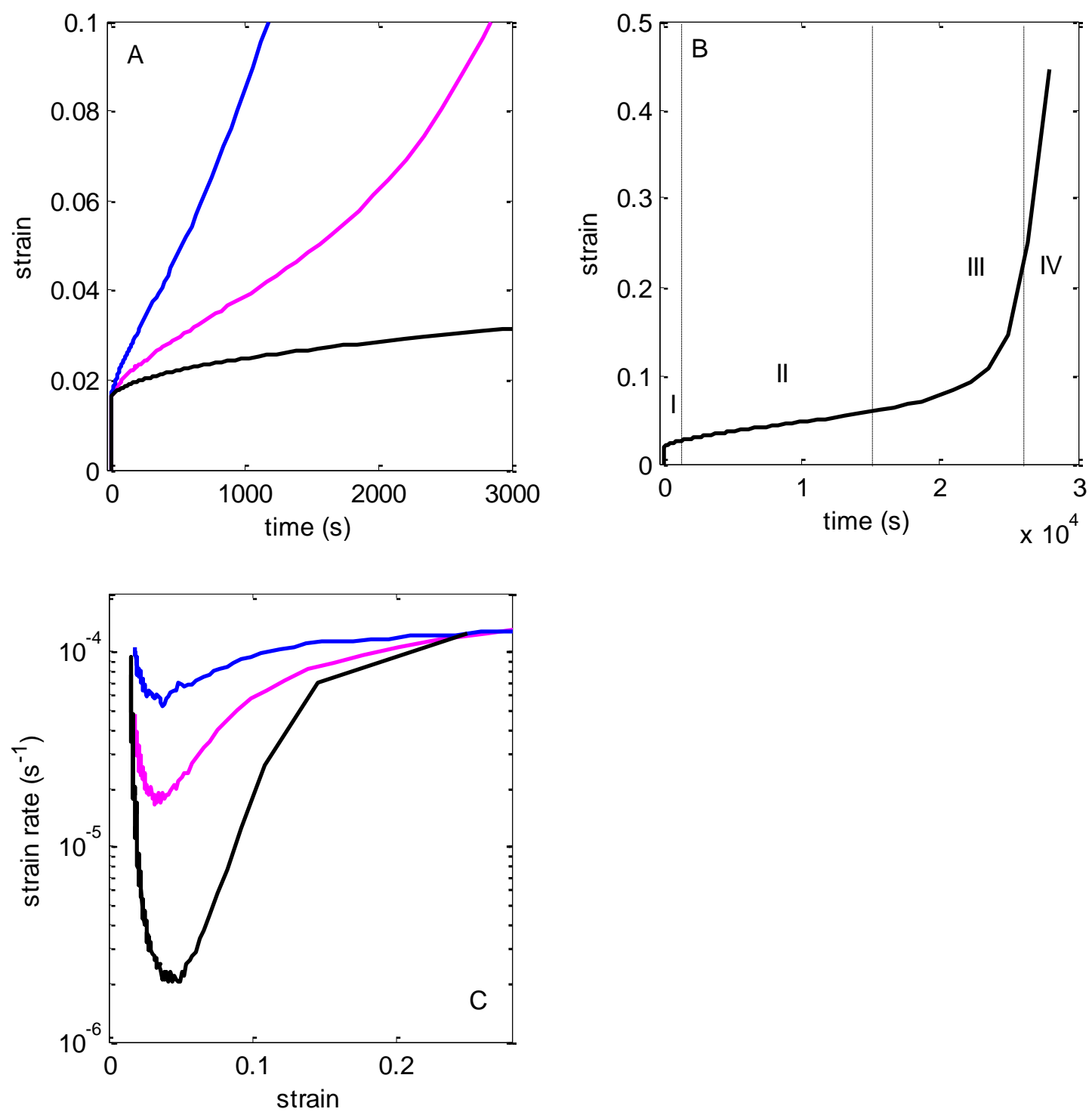

Figure 16 Effect of sub- $\mathrm{T}_{\mathrm{g}}$ aging on the nonlinear creep in uniaxial extension as predicted by the SCM. The applied stress is $17 \mathrm{MPa}$; material is cooled from $120^{\circ} \mathrm{C}$ at $1{ }^{\circ} \mathrm{C} / \mathrm{min}$ to $95^{\circ} \mathrm{C}$ where the creep simulation is carried out. Blue - aged for $0.2 \mathrm{~h}$ prior to creep; magenta - aged for $3 \mathrm{~h}$ prior to creep; black - equilibrated (i.e. aged for infinite time) prior to creep. (A) Strain vs time for short times; (B) strain vs time for the equilibrated material for longer times; (C) log strain rate vs strain plot.

One question remains. The creep can rapidly transition from primary creep to tertiary creep, with a very small region of secondary creep associated with the minimum in the strain rate vs. strain curve (see Fig. 10); alternatively, there can be an exceedingly long region of secondary creep as shown in Fig. 1. What is the origin of this significant difference in the length of time that the material exhibits secondary creep? This can be understood on the basis of examining the SCM predictions in Figs. 15 and 16. The fully equilibrated material (i.e. the black curve in Fig. 16) 
exhibits a long secondary creep period similar to the one shown in Fig. 1 as compared to the material aged for $0.2 \mathrm{~h}$ or $3 \mathrm{~h}$. In the schematics in Fig. 15, $\log \hat{a}$ pdf prior to deformation of the material aged for $0.2 \mathrm{~h}$ is shown as solid blue curve and $\log \hat{a}$ pdf prior to deformation of the equilibrated material as dashed blue curve. The long period of secondary creep for a fully equilibrated material corresponds to the transformation of the equilibrium $\log \hat{a}$ pdf (i.e. dashed blue curve) into the $\log \hat{a}$ pdf at the onset of tertiary creep (i.e. blue solid curve), which is very similar to the $\log \hat{a}$ pdf for a quenched material. What is the microstructural picture associated with this $\log \hat{a}$ pdf evolution in secondary creep? Comparing the $\log \hat{a}$ pdf of the equilibrated material vs. that of the quenched material, we observed that there are many more rigid domains for the equilibrated material. During the long secondary creep region for the equilibrated material, these rigid domains must transform into soft domains. As rigid domains are lost the strain must increase, because the stress is constant. Because the rigid-to-soft transition rate is to first order controlled by temperature and stress, which are both constant, the rate at which strain increases is also constant - i.e. secondary creep. This constant strain rate response only continues as long as the rigid domains dominate the mechanical response. Once the fraction of rigid domains is so depleted that it does not dominate the response, then the behavior reverts to tertiary creep that leads to Stage IV creep as discussed in the previous paragraphs.

\section{DISCUSSION}

The creep response of glassy polymers exhibit complex, nonlinear behavior, where this behavior provides an important perspective on the underlying relaxation processes. In addition to the wellknown linear viscoelastic creep behavior, glassy polymers exhibit as many as five stages creep. Of particular interest is tertiary creep, where the creep strain rate increases by as much as three orders-of-magnitude depending on the applied stress and thermal history. To-date, only a few constitutive models have reported the prediction of tertiary creep.

1. The Hasan-Boyce[7] and Drozdov et al.[8] models are representative of the class of models that postulate existence of internal variables, where the behavior of each of these variables is in turn governed by an evolution equation. The creep predictions by these models look good, but these models have difficulties predicting response under different experimental conditions. In addition, the internal variables have initial values that need to be set "by hand" depending on the thermal history prior to the mechanical experiment.

2. The EGP constitutive model[20] inextricably relates the tertiary creep to the same mechanism responsible for post-yield softening under constant strain rate, where EGP model produces qualitative predictions of the tertiary creep for a limited range of thermal histories. The key variable in the EGP model for tertiary creep and post-yield softening is the dependence of the plastic strain rate on the effective plastic strain, where the amount of stress overshoot has to be empirically set for each new thermal history, i.e. the EGP model just parameterizes the effect of thermal history on tertiary creep. 
3. The FLC constitutive model describes tertiary creep as well as creep-recovery from large deformations. The key variable in the FLC constitutive model [26] is the relaxation time, which is governed by an evolution equation that depends upon the strain rate. Because of the strain rate dependent relaxation time, the FLC model does not limit to linear viscoelasticity for small strains. In addition, the model parameters in the FLC model must change significantly in multi-step deformation histories like creep-recovery.

4. A modified version of the thermoviscoelastic constitutive model (TVEM) of Caruthers et al.[27] does predict tertiary creep in extension.[9] However, the TVEM predicts nearly an infinite increase in the rate of creep vs the finite increase observed experimentally. Moreover, with the same parameter set the TVEM does not predict tertiary creep in compression contrary to experiments. The TVEM can predict tertiary creep in compression for other thermal histories as a result of the intricate interplay of various relaxation integrals present in the $\log a$ functional. However, we believe that this sensitive dependence upon the details of the $\log a$ functional is a weakness of the TVEM model in light of the robust nature of tertiary creep observed experimentally.

Tertiary creep has proved a considerable challenge for constitutive modeling if the goal is not just curve-fitting, but rather a fundamental understanding of the underlying physical mechanism(s). The challenge is intensified when the constitutive model with the same parameters should also describe other deformation experiments. In summary, the existing constitutive models have serious deficiencies with respect to tertiary creep.

The traditional picture of nonlinear creep is that there are distinct regions of nonlinear creep. Early works on creep in plastics included mainly the characterization of primary and secondary creep.[6] However, under certain conditions the strain rate begins to accelerate and this region has been designated tertiary creep. Although the terms primary, secondary and tertiary creep are too embedded in the literature to now be changed, we believe that this is an unfortunate terminology, because it seems to imply that there are different mechanism at play in the various regions mechanisms that are incorporated in a number of the nonlinear viscoelastic/viscoplastic constitutive models. Examining both the data (e.g. Figs. 1-5, 8, and 10) as well as the predictions of the SCM (e.g. Figs. 12 and 16), we observe that there is a continuous evolution of the strain rate response during deformation. The identification of secondary creep is problematic as illustrated in Fig. 12A, since the definition of a 'constant' strain rate is arbitrary. In fact, the region of constant strain rate is only truly observed not prior but after tertiary creep as clearly shown in Figs. 4, 5, 8, and 10. The designation of primary, secondary and tertiary creep may provide some value as a phenomenological description of nonlinear creep behavior, but the identification with mechanisms is problematic.

The Stochastic Constitutive Model (SCM) previously reported[21] is able to naturally describe the various regions of the nonlinear creep response, i.e. primary, secondary and tertiary creep, as well as a new region of the creep strain response that we designate as Stage IV creep. Stage IV creep occurs after tertiary creep and is when the strain rate does become constant as has been 
experimentally observed for PMMA by Nanzai,[3] Hasan and Boyce,[7] and also in the present report as shown in Figs. 8 and 10. In several instances[1, 3-5] the creep rate at large strains then begins to decrease as the material begins to approach a strain plateau, where we designate this region of the creep response as Stage V creep. The SCM does not predict Stage V creep. Specific predictions of the SCM include:

1. Tertiary creep is predicted as the transition between the minimum in the $\log$ (strain rate) vs. strain curve and the constant strain rate response in Stage IV creep, where the magnitude of this transition increases with the applied stress (see Fig. 12) and sub-Tg annealing (see Fig. 16).

2. When the material is subject to the same thermal history, the SMC predicts that as the applied creep stress approaches the yield stress the time/strain range for which secondary creep is observed shrinks to the point that the material exhibits very little secondary creep (see Fig. 12)

3. The creep rate in the somewhat arbitrarily determined secondary creep region increases with stress (see Fig. 12) and decreases with aging time prior to deformation (see Fig. 16).

4. For small applied loads (e.g. the 7MPa predictions in Fig. 12B) the creep rate after the primary creep region is constant, i.e. no tertiary creep is predicted. This can be described as either (i) secondary creep that continues indefinitely, where the subsequent stages of creep never materialize or (ii) the Stage IV creep immediately follows primary creep, where the secondary and tertiary stages have been passed over.

5. The SCM predicts that the $\log a$ pdf has both a significant increase in the average mobility and a significant narrowing as parameterized by the increase in $\beta_{K W W}$ from 0.35 to 0.46 during tertiary creep (see Fig. 13A), predictions that are in qualitative agreement with the experimental molecular mobility measurements of Ediger and coworkers [5, 13, 38]. It should be pointed out, however, that the analysis by Ediger yields much bigger decrease in the relaxation time (up to three orders-of-magnitude) as well as much larger increase in $\beta_{K W W}$.

It is quite satisfying that although nothing has been added to the SCM for the case of nonlinear creep, it is able to qualitatively predict all the important features (with the exception of Stage $\mathrm{V}$ creep) of both the experimental creep data and the Ediger's molecular mobility experiments. Moreover, the SCM with the same parameters is able to describe, at least qualitatively, a variety of other nonlinear relaxation experiments including (i) nonlinear stress-strain behavior including post-yield softening,[21] (ii) the annealing time dependence of post-yield softening,[21] (iii) the inversion with the loading strain rate of the strain dependence of the rate of nonlinear stress relaxation,[23] (iv) stress memory,[18] and (v) the complex specific volume relaxation data of Kovacs.[22] We are unaware of any other constitutive model that is able to describe such a diverse set of relaxation phenomena.

The evolution of the $\hat{x}_{3}$ and $\log \hat{a}$ distributions in the SCM naturally describes the various stages of creep. First consider the linear viscoelastic response: 
1. Linear Viscoelastic Creep The linear viscoelastic creep is governed by the $\log \hat{a} \mathrm{pdf}$, where the application of an infinitesimal stress does not change this distribution. Specifically, the relaxation response of each of the meso-domains is described via a single Maxwell element, where the distribution of relaxation times seen in the linear viscoelastic compliance is due to the $\log \hat{a}$ distribution. The shape and position of the $\log \hat{a}$ pdf does depend upon the thermal history used to form the glass, where the effect of cooling rate and sub-Tg annealing on the linear viscoelastic creep is naturally accounted for by the evolution of the $\hat{x}_{0}$ pdf.

Now consider the various stages on nonlinear creep:

2. In Stage I - Primary Creep When the applied stress is more than infinitesimal, the rapid application of the load equally affects both rigid and mobile meso-domains, where the $\hat{x}_{3}$ distribution shifts to the left (i.e. the shift from the black to the violet curve in Fig. 13B). However, unless a large stress is applied, there is at first only a minimal change in the $\log \hat{a}$ pdf (Fig. 13A). Therefore even though the response is nonlinear during primary creep, the mechanism is essentially the same as in linear viscoelastic response. First, the strain is small and the elastically responding rigid domains alone cannot support the applied stress - the soft domains have to contribute which requires the strain rate to be fast. Then as strain continues to increase two processes begin to occur simultaneously: the soft domains relax and revert to a non-load supporting state and the remaining rigid domains support more and more of the load. This is clearly seen in Fig. 13B and 13D as a single-peak $\hat{x}_{3}$ pdf becomes a two-peak pdf, where the rigid meso-domains peak travels to the left and the 'soft' meso-domains peak centers on zero stress. The strain rate adjusts accordingly i.e. slows down. In fact if rigid meso-domains were permanently rigid the creep deformation would come to a full stop at the strain at which the elastic stress supported by the rigid meso-domains equals the applied stress. Of course this is not what actually happens, since (as explained above) the meso-domains identities are not permanent and there is a constant circulation where the rigid meso-domains stochastically become soft and vice versa. The presence of the applied stress affects the out-flux of rigid domains by making the conversion to soft more likely depending on how large a stress a given rigid meso-domain supports. This subtle influence has dramatic consequences, where the initial $\log \hat{a}$ pdf is only slightly shifted, but where it then begins to evolve resulting in emergence of subsequent stages of creep.

3. Stage II - Secondary Creep; Stage III - Tertiary Creep; Stage IV Creep These stages are better understood together as they are governed by the same underlying mechanism. Secondary and tertiary creep stages are associated with the substantial time evolution of the $\log \hat{a}$ pdf as shown in Figs. 12 and 16. Of critical importance is the location of the initial distribution (i.e. prior to application of the load or more precisely at the end of the primary creep, which is nearly the same) with respect to the location of the steady state distribution. This steady state is the Stage IV creep. At a given temperature the location of the steady state distribution and the associated strain rate is determined by the applied stress as discussed in Section V and shown schematically in Fig. 15, but not the aging time (see Fig. 16C where all aging times results in the same steady state strain rate). On the other hand, the location of the initial distribution is determined by thermal history, specifically the rate of cooling from above $\mathrm{T}_{\mathrm{g}}$ and the aging time at the temperature of the 
deformation experiment. Therefore two situations are possible (see Fig. 15): (i) the initial and the steady state distributions have roughly the same location or (ii) the initial distribution is located to the right of the steady state distribution. In case (i) Stages II, III, and IV of creep are indistinguishable from each other. In case (ii), however, there is some secondary creep at a slow rate followed by the transition stage i.e. the tertiary creep, which ends in the Stage IV creep at higher rate. Whereas the rate of the steady state i.e. Stage IV creep is fixed by the applied stress, the rate and the duration of the secondary creep depends critically on the location of the initial distribution and hence on the aging time. As explained in Section V, an aged material contains a significant excess of rigid domains (compare dashed vs solid blue curve in Fig. 15) and until this excess of rigid domains is fully expunged, the rate of creep remains slower than it would be in the Stage IV creep. This is manifested as the prolonged secondary creep observed in an aged sample. Conversely, in a quenched sample there is no excess fraction of "rigid" domains and the primary creep is immediately followed by a steady state creep at a constant strain rate.

4. Stage V Creep The slowing in growth, or even leveling off, of the axial strain with time as shown in Figs. 1 and 2 is not predicted by the SCM, where an additional physical mechanism that is not currently part of the SCM will be needed to describe Stage V creep. Alternatively, Stage V creep has only been observed in two experiments that are both technically difficult, where additional experimental data are needed to firmly establish the Stage V creep is a common feature of nonlinear creep of glassy polymers.

In summary, the SCM predicts the various stages of nonlinear creep, where the evolution of the $\hat{x}_{3}$ distribution and the $\log \hat{a}$ distribution is what controls the rich nonlinear relaxation behavior.

The predictions of SCM are consistent with the postulate that post-yield softening in a constant strain rate deformation is related the 'softening' that occurs during tertiary creep.[3] Other constitutive models (e.g. EGP[4, 20]) also are consistent with this postulate; however, these models are unable to predict the effect of thermal history on either the post-yield softening and/or tertiary creep, where the effect of thermal history on the critical softening must be determined experimentally for each new thermal history. In contrast, the SCM predicts the post-yield stress softening in constant strain rate experiments and tertiary creep as well as their dependence on thermal history. The SCM mechanism for the tertiary creep is based on the fact that the distribution of the local relaxation times in the material (i.e. the distribution of 'rigid' and 'soft/mobile' domains) immediately after the application of the load evolves in a complex manner during nonlinear creep. The shape of the relaxation distribution changes with time, i.e. the relaxation spectrum is not thermorheologically simple (or more precisely the material is not timeshift invariant). Specifically, the SCM predicts that during creep some of the load-bearing rigid meso-domains become non-load-bearing mobile meso-domains. The ensemble average of the meso-domain stress distribution is the macroscopic axial stress, which is constant in a creep deformation; thus, the stress on remaining load bearing meso-domains will increase, where the dynamic process of inter-conversion between rigid and mobile meso-domains contained in the SCM gives rise to the evolution of stress distribution of the meso-domains and the associated shape of the relaxation spectra. 
In summary, the SCM is able to (i) at least qualitatively predict the major features of nonlinear creep including tertiary creep and (ii) provides a precise mechanistic picture of the origins of the various regions of nonlinear creep. The SCM in its current form does have one limitation with respect to nonlinear creep - it does not predict Stage V creep as illustrated in Fig. 1.

\section{ACKNOWLEDGEMENTS}

This work was supported by National Science Foundation Grant Number 1363326-CMMI. 


\section{APPENDIX A. Traditional Constitutive Models}

In order to not interrupt the flow of the main text, the detailed description of the various constitutive models discussed in this paper has been deferred to this appendix.

\section{A.1 Hasan and Boyce Viscoplastic Constitutive Model}

The Hasan-Boyce model[7] is described by the following equations (where we have changed notation somewhat in order to be consistent with the notation used throughout this paper and corrected a number of typos in the original paper). The one-dimensional form of the stress-strain relationship is given by

$$
\frac{1}{\mu} \dot{\sigma}_{12}=\dot{\gamma}-\dot{\gamma}_{p}
$$

where $\sigma_{12}$ is the shear stress, $\gamma$ is the total shear strain, and $\gamma_{p}$ is the plastic shear strain; $\mu$ is the glassy shear modulus. As the authors point out, Eqn. (A.1) can be easily modified to describe uniaxial deformations instead of shear. The plastic flow rule is given by Eqn. (2) in the main text and the function $y(x)$ is

$$
y(x)=\frac{1}{2 \mathrm{e}^{\frac{3 \pi}{4}}+\sqrt{2}}\left\{\frac{(2-x) e^{(1-x) \frac{3 \pi}{4}}+\sqrt{2}}{1+(1-x)^{2}}+\frac{(2+x) e^{(1-x) \frac{3 \pi}{4}}+e^{-x \frac{3 \pi}{2}} \sqrt{2}}{1+(1+x)^{2}}\right\}
$$

The evolution equations for the three internal variables $\tilde{a}, 1 / \tilde{\alpha}$, and $\tilde{s}$ are given by

$$
\begin{gathered}
\dot{\tilde{a}}=-\left(\tilde{a}-\tilde{a}_{e q}\right) \exp \left[-\varsigma e^{-\zeta \gamma_{p}}\right] \omega \\
\left(\frac{\dot{1}}{\tilde{\alpha}}\right)=-\left(\frac{1}{\tilde{\alpha}}-\frac{1}{\tilde{\alpha}_{e q}}\right) \omega \\
\dot{\tilde{s}}=-\left\{\tilde{s}-\frac{\sigma_{12}}{\sigma_{0}} \tilde{s}_{0}\left[1+\beta_{2} e^{-\beta_{3} \gamma_{p}}\right]\right\} \omega
\end{gathered}
$$

where $\omega$ is frequency given by Eqn. (27) in [7] and $\tilde{a}_{e q}, 1 / \tilde{\alpha}_{e q}, s_{0}, \varsigma, \beta_{2}$, and $\beta_{3}$ are model parameters. The initial conditions $\tilde{a}(t=0), \tilde{\alpha}(t=0)$ and $\tilde{s}(t=0)$ all depend on the thermal history prior to deformation and must be set by hand; hence, they are fitting parameters. Because the frequency $\omega$ cannot be negative, the evolution equations are first-order ODEs; thus, Eqns. (A.3) and (A.4) result in a monotonic approach to the equilibrium values of $\tilde{a}_{e q}$ and $1 / \tilde{\alpha}_{e q}$. However, in Figs. 14a and $14 \mathrm{~b}$ in [7] the quantities $\tilde{a}$ and $1 / \tilde{\alpha}$ exhibit non-monotonic behavior 
upon loading followed by unloading. This inconsistency concerning the evolution of the internal variables needs to be resolved in order to accept the constitutive predictions reported for the Hasan and Boyce model.

\section{A.2 Thermoviscoelastic Constitutive Model}

The thermoviscoelastic model (TVEM)[27, 39] extends the rational mechanics framework of Coleman[40] to include relaxation on a material time $t^{*}$. The TVEM model has been simplified (and modified) by Adolf et al.,[9] where the resulting model was designated the SPEC model, which stands for the Simplified Potential Energy Clock model. Tertiary creep predictions were made using the SPEC version of TVEM, where the creep predictions by the full TVEM have not been unexplored. The SPEC stress constitutive equation is given by Eqn. (7) in the main text. The expression in Eqn. (7) only includes the single integral contributions, where the complete TVEM expression through second order terms is given in [27]. The key nonlinearity in both TVEM and SPEC models is contained in the material clock $t^{*}$ defined in Eqn. (8), where $a(\mathrm{t})$ is a functional of the thermal and deformation history. In the SPEC model the $a(\mathrm{t})$ is assumed to be given by:

$$
\log a=C_{1}\left(\frac{C_{2}^{\prime \prime}}{C_{2}^{\prime \prime}+\Delta}-1\right)
$$

where

$$
\begin{aligned}
\Delta= & \theta-\theta_{r e f}-\int_{-\infty}^{t} d s f_{C}\left(t^{*}-s^{*}\right) \frac{d \theta}{d s}+C_{3}\left[I_{1}-\int_{-\infty}^{t} d s f_{A}\left(t^{*}-s^{*}\right) \frac{d I_{1}}{d s}\right] \\
& +C_{4} \int_{-\infty}^{t} \int_{-\infty}^{t} d s d u f_{G}\left(t^{*}-s^{*}, t^{*}-u^{*}\right) \frac{d \mathbf{H}^{\prime}}{d s}: \frac{d \mathbf{H}^{\prime}}{d u}
\end{aligned}
$$

The coefficients $C_{1}, C_{2}^{\prime \prime}, C_{3}$, and $C_{4}$ are model parameters in SPEC unlike in TVEM where they are expressed in terms of material properties as defined in Eqns. (B.5-8). The relaxation functions $f_{C}(t), f_{A}(t)$ and $f_{G}(t)$ are the normalized viscoelastic relaxation functions associated respectively with the constant volume heat capacity, thermal stress and shear modulus. There are multiple ways to compute the double integral contributions to the TVEM.[27] If the memory kernel in the double integral in Eqn. (A.7) is a product of two $f_{G}(t)$ functions, then Eqns. (7) and (A.6-7) result in a 'stress clock' model, where there is no dependence on the deviatoric component of the strain in $t^{*}$. Such a version of the TVEM has no hope of describing tertiary creep, because the stress contribution to $\log a$ is a constant and thus will not decrease with time as required for the acceleration of relaxation associated with tertiary creep. Alternatively, if the memory kernel in Eqn. (A.7) is not factorizable, then the SPEC $\log a$ does contain dependence on deviatoric strain history. The non-factorizable form of the memory kernel used by Adolf et al.[9] when they described tertiary creep is given by (i.e. Case 1 in [27]) is given by 


$$
f(t, s)=\sum_{i} A_{i} \exp \left[-\frac{t+s}{\tau_{i}}\right]
$$

where $\operatorname{set}\left\{\tau_{i}, A_{i}\right\}$ is a Prony series representation of a KWW relaxation function with a given $\beta_{K W W}$ and $\tau_{K W W}$. The KWW parameters for each of the memory kernels $f_{C}(t), f_{A}(t)$ and $f_{G}(t)$ in SPEC are optimized to fit the data.[9]

The presence of the history integrals in the $\log a$ functional creates complicated feedback loops that strongly affect the response of the material to various thermo-mechanical stimuli. For example, an increase in the strain rate results in a larger $\Delta$ via Eqn. (A.7) and hence smaller $\log a$ due to Eqn. (A.6); which causes more rapid relaxation, where this rapid relaxation in turn causes the integrals in Eqn. (A.7) to decay faster; which then causes $\Delta$ to become event smaller; and so forth, in a complex feedback loop. The reason for the absence of strain rate singularity in compression is a stabilization of $\log a$ as illustrated in Fig. 5b. The double integral term in Eqn. (A.7) is always positive, where its increase results in the higher rate of relaxation. This higher rate of relaxation causes the integrals in the stress equation Eqn. (7) to decay faster. Thus, to maintain the stress constant during creep the strain rate has to increase - this is the positive feedback mechanism, which is active in both extension and compression. To understand the difference between extension and compression response one has to examine the volume term in $\log a-$ i.e. the term in Eqn. (A.7) containing the $C_{3}$ factor, which is plotted in Fig. A.1. Negative values of this term result in lower values of $\Delta$ and hence slower relaxation and the positive values more rapid relaxation. It is clear that in compression this term compensate the effects of the double integral term resulting in near constant $\log a$. Note that in case of extension this volume integral term also eventually turns negative, but by that time the effect of the double integral term dominates. This analysis demonstrates that the balance between various terms in SPEC $\log a$ can be quite delicate and hence strongly dependent on the thermo-deformational history. It should be emphasized again that these predictions in Figs. 5 and A.1 are for particular thermal histories studied in [9]. Under different thermal histories the predictions change, e.g. the SPEC does predict tertiary creep in compression in case of a sample equilibrated at $55^{\circ} \mathrm{C}$ (i.e. $\theta_{g}-15^{\circ} \mathrm{C}$ ), which would require an exceedingly long annealing time. Perhaps readjusting the material parameters or even their functional form would allow the TVEM to describe tertiary creep in both extension and compression for the full range of thermal histories. However it is quite worrisome that the emergence of the tertiary creep behavior in TVEM is so sensitive to the thermodeformation history (i.e. switching from a nearly singular response to no tertiary creep at all), while experimentally the presence of tertiary creep is a very robust observation. 


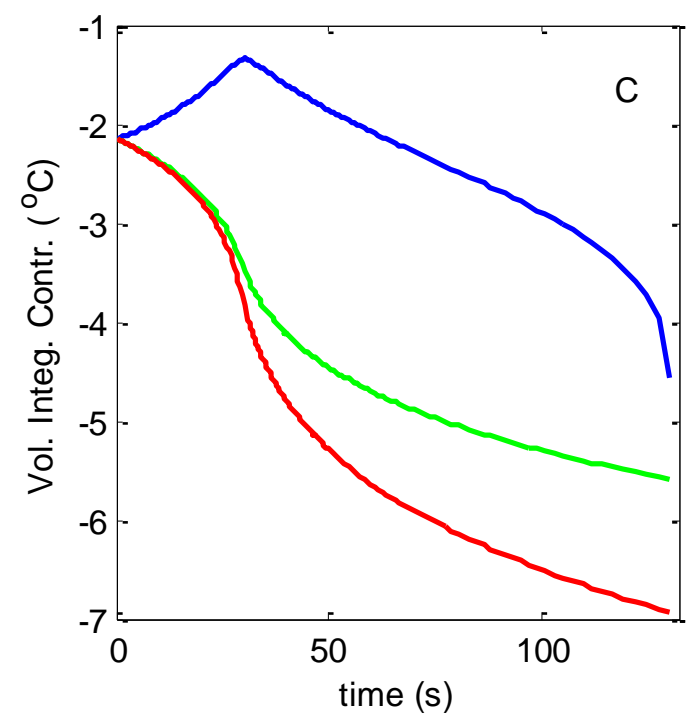

Figure A.1 (Notation is the same as in Figs. 6A and 6B in the main text) Volume integral contribution to $\log a$ in the SPEC model (see formula in the text) during creep. SPEC model parameters same as in [9] for the DGEBA/DEA epoxy resin.

\section{APPENDIX B. Stochastic Constitutive Model}

In this appendix we will summarize key features of the Stochastic Constitutive Model (SCM) developed in [21] in order to enable the interpretation of the various internal variables for the case of nonlinear creep of interest in this paper. By assumption the mesoscopic $\log \hat{a}$ shift factor is controlled by (i) the macroscopic temperature and strain that is common to all domains in the ensemble and (ii) the fluctuating entropy and stress that varies from domain to domain (see Eqns. 55 to 59 in [21]). Fluctuating quantities are denoted with a 'hat'. Specifically, it is assumed that mesoscopic mobility $\hat{a}$ controlled by the configurational internal energy $\hat{e}_{c}$ according to

$$
\log \hat{a}=c_{1}\left(\frac{e_{c R}}{\hat{e}_{c}}-1\right)
$$

where $e_{c R}$ is the configurational internal energy in the reference state and $c_{1}$ is the WLF constant. $\hat{e}_{c}$ depends upon seven stochastic state variables $\hat{x}_{i}, i=0,6$ corresponding to entropy $(i=0)$ and the six components of the stress tensor

$$
\hat{e}_{c}=e_{c}^{\infty}(\theta, V)+\theta_{R} \sigma_{S} \hat{x}_{0}+\frac{1}{4} \frac{V_{R} \sigma_{G}^{2}}{\Delta G}\left[\hat{x}_{2}^{2}+\hat{x}_{3}^{2}+2\left(\hat{x}_{4}^{2}+\hat{x}_{5}^{2}+\hat{x}_{6}^{2}\right)\right]
$$

The equilibrium configurational internal energy is given by 


$$
e_{c}^{\infty}=e_{c R}+\theta_{R} 2 \Delta A \frac{V-V_{R}}{V_{R}}+2 \theta_{R} \Delta C\left(\theta-\theta_{R}\right)
$$

where

$$
e_{c R}=c_{2}\left(2 \Delta A \alpha_{\infty} \theta_{R}+2 \Delta C \theta_{R}\right)
$$

$\theta_{R}$ and $V_{R}$ are respectively the temperature and the specific volume in the reference state and $c_{2}$ is the WLF constant. The material parameters in Eqns. (B.2-4) are determined from the limiting behavior in the glassy and rubbery/melt states

$$
\begin{gathered}
\Delta G=G_{g}-G_{\infty} \\
\Delta K=K_{g}-K_{\infty} \\
2 \Delta A=-V_{R}\left[K_{g} \alpha_{g}-K_{\infty} \alpha_{\infty}\right] \\
2 \Delta C=V_{R}\left[K_{g} \alpha_{g}^{2}-K_{\infty} \alpha_{\infty}^{2}\right]-\frac{1}{\theta_{R}}\left[C_{p g}\left(\theta_{R}\right)-C_{p \infty}\left(\theta_{R}\right)\right]
\end{gathered}
$$

where $G$ is the shear modulus, $K$ is the bulk modulus, $\alpha$ is the coefficient of thermal expansion, and $C_{p}$ is the constant pressure heat capacity; the subscripts ' $\mathrm{g}$ ' and ' $\infty$ ' stand for glassy and equilibrium (melt) state, respectively. The magnitudes of the fluctuations in entropy, dilatational stress (i.e. pressure), and deviatoric stress are given by

$$
\sigma_{S}^{2}=\frac{1}{L^{3}} V k_{B} C_{p g} \quad \sigma_{K}^{2}=\frac{1}{L^{3}} 2 k_{B} \theta \frac{C_{p g}}{C_{V g}} 3 K_{g} \quad \sigma_{G}^{2}=\frac{1}{L^{3}} 2 k_{B} \theta \frac{C_{p g}}{C_{V g}} 2 G_{g}
$$

where $L$ is the size of the meso-domain and $C_{V}$ is the constant volume heat capacity. The fluctuating variables that control local relaxation rate are defined as

$$
\begin{gathered}
\hat{x}_{0}=\frac{\hat{S}-S_{\infty}}{\sigma_{S}} \\
\hat{x}_{1}=\frac{1}{\sqrt{3} \sigma_{K}}\left(\left(\hat{\tilde{T}}_{\mathbf{H} 11}-\tilde{T}_{\mathbf{H} 11}^{\infty}\right)+\left(\hat{\tilde{T}}_{\mathbf{H} 22}-\tilde{T}_{\mathbf{H} 22}^{\infty}\right)+\left(\hat{\tilde{T}}_{\mathbf{H} 33}-\tilde{T}_{\mathbf{H} 33}^{\infty}\right)\right) \\
\hat{x}_{2}=\frac{1}{\sqrt{2} \sigma_{G}}\left(\left(\hat{\tilde{T}}_{\mathbf{H} 22}-\tilde{T}_{\mathbf{H} 22}^{\infty}\right)-\left(\hat{\tilde{T}}_{\mathbf{H} 33}-\tilde{T}_{\mathbf{H} 33}^{\infty}\right)\right)
\end{gathered}
$$




$$
\begin{gathered}
\hat{x}_{3}=\frac{1}{\sqrt{6} \sigma_{G}}\left(-2\left(\hat{\tilde{T}}_{\mathbf{H} 11}-\tilde{T}_{\mathbf{H} 11}^{\infty}\right)+\left(\hat{\tilde{T}}_{\mathbf{H} 22}-\tilde{T}_{\mathbf{H} 22}^{\infty}\right)+\left(\hat{\tilde{T}}_{\mathbf{H} 33}-\tilde{T}_{\mathbf{H} 33}^{\infty}\right)\right) \\
\hat{x}_{4}=\frac{1}{\sigma_{G}}\left(\hat{\tilde{T}}_{\mathbf{H} 12}-\tilde{T}_{\mathbf{H} 12}^{\infty}\right) \quad \hat{x}_{5}=\frac{1}{\sigma_{G}}\left(\hat{\tilde{T}}_{\mathbf{H} 13}-\tilde{T}_{\mathbf{H} 13}^{\infty}\right) \quad \hat{x}_{6}=\frac{1}{\sigma_{G}}\left(\hat{\tilde{T}}_{\mathbf{H} 23}-\tilde{T}_{\mathbf{H} 23}^{\infty}\right)
\end{gathered}
$$

Here $S$ is the entropy and $\tilde{\mathbf{T}}_{\mathbf{H}}$ is the stress tensor conjugate to the Hencky strain tensor $\mathbf{H}$.

The dynamic equations for the fluctuating entropy and the six component of (symmetric) stress tensor are given in Eqns. 52 and 53 in [21]

$$
\begin{gathered}
d \hat{x}_{0}=d \hat{r}_{0}-\frac{2 \Delta C}{\sigma_{S}} d \theta-\frac{2 \Delta A}{\sigma_{S}} \frac{d V}{V_{R}} \\
d \hat{x}_{1}=d \hat{r}_{1}+\sqrt{3} \frac{\Delta K}{\sigma_{K}} \frac{d V}{V_{R}}+\sqrt{3} \frac{2 \Delta A}{\sigma_{K} V_{R}} d \theta \\
d \hat{x}_{2}=d \hat{r}_{2}+\frac{2 \Delta G}{\sigma_{G}}\left(d H_{22}-d H_{33}\right) \frac{1}{\sqrt{2}} \\
d \hat{x}_{3}=d \hat{r}_{3}+\frac{2 \Delta G}{\sigma_{G}}\left(-2 d H_{11}+d H_{22}+d H_{33}\right) \frac{1}{\sqrt{6}} \\
d \hat{x}_{4}=d \hat{r}_{4}+\frac{2 \Delta G}{\sigma_{G}} d H_{12} \quad d \hat{x}_{5}=d \hat{r}_{5}+\frac{2 \Delta G}{\sigma_{G}} d H_{13} \quad d \hat{x}_{6}=d \hat{r}_{6}+\frac{2 \Delta G}{\sigma_{G}} d H_{23}
\end{gathered}
$$

where the stochastic terms are

$$
d \hat{r}_{n}=-\frac{d t}{a \tau_{0}}\left[\hat{x}_{n}+\frac{\partial}{\partial \hat{x}_{n}} \ln (a)\right]+\frac{1}{\sqrt{a \tau_{0}}} \sqrt{2} d \hat{W}_{n} \quad n=0, \ldots, 6
$$

Mathematically these are the stochastic differential equations (SDE) that include: (i) a single relaxation time Debye process (i.e. the first term in square brackets on the right hand side of Eqn. (B.20)); (ii) a Wiener processes $\mathbf{d} \hat{\mathbf{W}}$ that captures the effect of the inherent fluctuations present in a small system; and (iii) an additional drift term (i.e. the second term in square brackets on the right hand side of Eqn. (B.20)) needed to satisfy the requirement that the equilibrium distribution be Gaussian. Each of the defining nonlinear stochastic equations (i.e. Eqns. (52) and (53) in [21]) consists of a stochastic part and a non-stochastic part that contains the effects of instantaneous changes in the macroscopic temperature and strain tensor. The stochastic constitutive model describes the fluctuating stress and entropy response to a given deformation and temperature 
history. Finally, the boundary conditions of the problem are an ensemble average of multiple realizations of Eqns. (B.15-20) given by

$$
\tilde{\mathbf{T}}_{\mathbf{H}}=\left\langle\hat{\tilde{\mathbf{T}}}_{\mathbf{H}}\right\rangle
$$

In case of a uni-axial deformation, Eqn. (9) in the main text follows from Eqn. (B.21). 


\section{REFERENCES}

1. $\quad$ Ender DH. Journal of Applied Physics 1968;39(11):4877-4882.

2. $\quad$ Ender DH. Journal of Macromolecular Science, Part B: Physics 1970;4(3):635-648.

3. Nanzai Y. JSME International Journal, Series A 1994;37(2):149-154.

4. Klompen ETJ, Engels TAP, van Breemen LCA, Schreurs PJG, Govaert LE, and Meijer HEH. Macromolecules 2005;38(16):7009-7017.

5. $\quad$ Lee H-N, Paeng K, Swallen SF, and Ediger MD. Science 2009;323:231-234.

6. Findley WN, Lai JS, and Onaran K. Creep and relaxation of nonlinear viscoelastic materials. New York: Dover Publications, Inc., 1989.

7. Hasan OA and Boyce MC. Polymer Engineering and Science 1995;35(4):331-344.

8. Drozdov AD, Hog Lejre A-L, and Christiansen Jd. Composites Science and Technology 2009;69:2596-2603.

9. Adolf DB, Chambers RS, and Neidigk MA. Polymer 2009;50:4257-4269.

10. Ediger MD. Private communication.

11. Knauss WG and Zhu W. Mechanics of Time-Dependent Materials 2002;6:231-269.

12. Turner S. Creep in glassy polymers. In: Haward RN, editor. The physics of glassy polymers. London: Applied Science Publishers, Ltd, 1973. pp. 223-277.

13. Lee H-N and Ediger MD. Journal of Chemical Physics 2010;133:014901.

14. Ferry JD. Viscoelastic Properties of Polymers, Third ed. New York: John Wiley \& Sons, 1980.

15. Kovacs AJ. Fortschritte der Hochpolymeren-Forschung 1963;3:394-507.

16. Bowden PB. The yield behavior of glassy polymers. In: Haward RN, editor. The physics of glassy polymers. London: Applied Science Publishers Ltd, 1973. pp. 279-339.

17. Haward RN. The post-yield behaviour of amorphous plastics. In: Haward RN, editor. The physics of glassy polymers. London: Applied Science Publishers, Ltd, 1973. pp. 340-393.

18. Kim JW, Medvedev GA, and Caruthers JM. Polymer 2013;54(21):5993-6002.

19. Ames NM, Srivastava V, Chester SA, and Anand L. International Journal of Plasticity 2009;25:1495-1539.

20. Klompen ETJ, Engels TAP, Govaert LE, and Meijer HEH. Macromolecules 2005;38(16):6997-7008.

21. Medvedev GA and Caruthers JM. Journal of Rheology 2013;57(3):949-1002.

22. Medvedev GA and Caruthers JM. Macromolecules 2015;48(3):788-800.

23. Medvedev GA, Kim JW, and Caruthers JM. Polymer 2013;54(24):6599-6607.

24. Leaderman H, McCrackin F, and Nakada O. Transactions of The Society of Rheology (1957-1977) 1963;7:111-123.

25. Green AE and Rivlin RS. Archive for Rational Mechanics and Analysis 1957;1:1-21.

26. Fielding SM, Larson RG, and Cates ME. Physical Review Letters 2012;108:048301.

27. Caruthers JM, Adolf DB, Chambers RS, and Shrikhande P. Polymer 2004;45:4577-4597.

28. Chen K and Schweizer KS. Physical Review E 2010;82:041804.

29. Kontou E and Spathis G. Mechanics of Time-Dependent Materials 2014;18:373-386.

30. Haward RN and Thackray G. Proceedings of the Royal Society of London. Series A, Mathematical and Physical Sciences 1968;302(1471):453-472.

31. Dreistadt C, Bonnet AS, Chevrier P, and Lipinski P. Materials and Design 2009;30:31263140.

32. Schapery RA. Polymer Engineering and Science 1969;9(4):295-310.

33. Knauss WG and Emri I. Polymer Engineering and Science 1987;27(1):86-100. 
34. Popelar CF and Liechti KM. Journal of Engineering Materials and Technology 1997;119(3):205-210.

35. Chen K and Schweizer KS. Macromolecules 2011;44:3988-4000.

36. Lee EW, Medvedev GA, and Caruthers JM. Journal of Polymer Science: Part B: Polymer Physics 2010;48(22):2399-2401.

37. Ediger MD. Annual Review of Physical Chemistry 2000;51:99-128.

38. Lee H-N, Paeng K, Swallen SF, Ediger MD, Stamm RA, Medvedev GA, and Caruthers JM. Journal of Polymer Science: Part B: Plymer Physics 2009;47:1713-1727.

39. Lustig SR, Shay RMJ, and Caruthers JM. Journal of Rheology 1996;40(1):69-106.

40. Coleman BD and Noll W. Reviews of Modern Physics 1961;33:239-249. 